\title{
Regenerating CNS myelin - from mechanisms to experimental medicines
}

Robin J. M. Franklin ${ }^{1}$ and Charles ffrench-Constant ${ }^{2}$

${ }^{1}$ Wellcome Trust-Medical Research Council Cambridge Stem Cell Institute, Clifford Allbutt Building, Cambridge Biomedical Campus, University of Cambridge, Cambridge CB2 OAH, UK

${ }^{2} M R C$ Centre for Regenerative Medicine, Edinburgh bioQuarter, University of Edinburgh, Edinburgh, EH16 4UU, UK

rjf1000@cam.ac.uk

cffc@ed.ac.uk

Key points:

- Remyelination is a spontaneous regenerative process in the adult mammalian central nervous system in which new oligodendrocytes and myelin sheaths are generated from a widespread population of adult progenitor cells.

- Remyelination involves the distinct stages of progenitor activation, recruitment (proliferation and migration) and differentiation into mature myelin-sheath forming oligodendrocytes: each is orchestrated by a complex network of cells and signaling molecules.

- The efficiency of remyelination declines progressively with adult aging, a phenomenon that has a profound bearing on the natural history chronic demyelinating diseases such as multiple sclerosis, although experimental studies have revealed that the age-affects are reversible.

- Remyelination is neuroprotective, limiting the axonal degeneration that follows demyelination. Restoring remyelination is therefore an important therapeutic goal so as prevent neurodegeneration and progressive disability in multiple sclerosis and other myelin diseases.

- Insights into the mechanism governing remyelination as well as an increasing number of high throughput screening platforms have led to the identification of a number of drug targets for the pharmacological enhancement of remyelination, some of which have entered clinical trials.

- Advances in the generation of large numbers of human stem and progenitor cells, coupled with compelling preclinical data, have opened up new opportunities for cell based remyelination therapies, especially for the leucodystrophies. 


\section{Author biographies}

Robin Franklin is Professor of Stem Cell Medicine at the Wellcome Trust-Medical Research Council Cambridge Stem Cell Institute and is Director of the UK MS Society Cambridge Centre for Myelin Repair at the University of Cambridge. He obtained his undergraduate degrees in Physiology and Veterinary Medicine at the University of London and his PhD in Neuroscience at the University of Cambridge. His main research questions are how do stem cells in the adult brain respond to injury, how do they contribute to regeneration, and how are they are affected by ageing?

Charles ffrench-Constant is a Professorial Fellow, co-director of the MS Society Edinburgh Centre of MS Research, and Dean of Research for the College of Medicine and Veterinary Medicine at the University of Edinburgh. He was director of the MRC Centre for Regenerative Medicine in Edinburgh from 2011-2016. He obtained his undergraduate degrees in physiology and in medicine from Cambridge University and University College London, and his PhD in Neuroscience from University College London. His research interest is the developmental and cell biology of oligodendrocytes and myelination, and the overlaps between myelin development and regeneration. 
Abstract | Although the core concept of remyelination - based on the activation, migration, proliferation and differentiation of CNS progenitors - has not changed over the past 20 years, our understanding of the detailed mechanisms that underlie this process has developed considerably. We can now decorate the central events of remyelination with a host of pathways, molecules, mediators and cells, revealing a complex and precisely orchestrated process. These advances have led to recent drug-based and cell-based clinical trials for myelin diseases, and have opened up hitherto unrecognized opportunities for drug-based approaches to therapeutically enhance remyelination. 
Contrary to the oft-expressed view that the CNS has little capacity for regeneration, injury to oligodendrocytes, the myelin-forming cells of the CNS, can be followed by a robust regenerative response leading to the formation of new myelin sheaths - a process termed remyelination ${ }^{1,2}$. This regenerative response is most clearly seen in young animals following experimental demyelinating lesions, which can be created by a number of techniques (BOX 1). It is also seen in humans following lesions such as those caused by the demyelinating disease multiple sclerosis $(\mathrm{MS})^{3-5}$. For many patients with this disease, however, remyelination ultimately fails and it is thought that the loss of metabolic support normally provided by myelin sheaths to axons contributes to the axonal and neural degeneration and to the progressive disability that characterise the later stage of $\mathrm{MS}^{6,7}$.

The study of remyelination is important to biologists and clinicians alike, as it provides an excellent exemplar of an important and emerging discipline - tissue regeneration. The inexorable rise in disability within ageing populations represents one of the major challenges for health care in the 21st-century, yet there are no therapies in the clinic that directly address this by promoting tissue regeneration - all such treatments simply prevent further damage (although in so doing they may allow natural healing events to proceed more efficiently). When the endogenous regenerative capacity becomes limited, as in individuals with MS, disability inevitably results. What is therefore required is an understanding of tissue regeneration - what drives it and why does it fail?

A key question in tissue regeneration is how, against a backdrop of the potentially hostile environment of damaged tissue that has attained its full size and complexity, can new cells generated from residents stem and progenitor populations integrate and become functional? The study of remyelination provides an accessible process to study these issues. It is also a very important area of research in its own right; if, as we will argue below, remyelination is neuroprotective and can be enhanced in the CNS following diseases characterised by myelin loss, such as MS and perinatal white-matter injury, effective therapies for diseases that impose an enormous financial burden on society become a realistic goal.

As we stated in our previous reviews in 2002 and 2008, the major challenge for the field remains the discovery and delivery into the clinic of drugs that enhance remyelination and lessen neurodegeneration ${ }^{1,2}$. Since 2008 , however, there has been a step change in our understanding of the cells and the signalling pathways that are responsible for remyelination, on a scale that now defies comprehensive coverage in a single review. Here, we review some of the advances that have occurred, focusing on how the underlying biology has provided a platform for the identification of biologics and small molecules that enhance remyelination and are heralding the advent of a new experimental medicine-based era.

\section{[H1] Why is remyelination important?}

CNS myelin has two functions: it provides metabolic support to the axon and allows rapid transmission of action potentials along the axon ${ }^{6,8,9}$. In the former, monocarboxylate transporters on the oligodendrocyte enable the transfer of lactate from the glial cell to the axon and, in doing so, provide the substrate for axonal ATP production via the citric acid cycle ${ }^{10-12}$. In the latter, nodes of Ranvier form by adhesive interactions between axon and 
paranodal loops at the end of each sheath, leading to the localisation of voltage-dependent sodium channels in the gaps between sheaths, which enables saltatory conduction ${ }^{13,14}$. The rationale for remyelination therapies is therefore that they will both restore metabolic support to the axon to prevent the axon degeneration responsible for progressive disability and restore the nodes that are required to facilitate conduction and hence function (FIG. 1). Below, we examine the experimental evidence supporting these objectives.

[H3] Prevention of neurodegeneration. If remyelination prevents axon degeneration, CNS regions in which remyelination is enhanced should show increased numbers of viable axons. Indeed, various human and animal neuropathological studies suggest that axonal degeneration occurs more in areas of acute and chronic demyelination ${ }^{15}$ than in areas of remyelination ${ }^{16}$. However, such studies do not show causality - successful remyelination might simply reflect the presence of healthy axons that are able to support new myelin formation, while remyelination failure might result from axonal damage perturbing any physical and biochemical cues required for myelination. The need to consider this alternative explanation is highlighted by the evidence for intrinsic axonal defects, such as mitochondrial abnormalities, as potential causes of axonal and neuronal degeneration in MS ${ }^{17-19}$.

Distinguishing cause and effect requires experimentation, and can only be addressed in animal models in which the strategy used to prevent or enhance remyelination has no direct effect on the axons. Three experimental strategies have been used to address this issue. The first has been the transplantation of cells capable of remyelination after administration of the oligodendrocyte toxin cuprizone to mice combined with the use of irradiation to prevent endogenous remyelination. This approach rescued remyelination and led to a decrease in axonal damage ${ }^{20}$. The second strategy has been the selective genetic ablation of oligodendrocytes by the cell-specific expression of diphtheria toxin to induce demyelination which resulted in secondary axonal injury, an effect that was still observed even when the activation of the adaptive immune system, which could lead to bystander damage of axons, was prevented ${ }^{21}$. The third strategy has involved enhancing remyelination by the removal the M1 muscarinic receptor from oligodendrocytes so as to enhance their differentiation. This approach showed increased preservation of axons in an experimental autoimmune encephalomyelitis $(E A E)^{22}$. Although these studies do not completely rule out the possibility that immunomodulatory effects of progenitors on the microglia or other cells types within the lesion may contribute to axonal injury or protection, together they do provide persuasive evidence for a direct neuroprotective effect of remyelination.

[H3] Restoration of function. The effectiveness of remyelination in restoring conduction velocity is well established. Electrophysiological studies in the rodent spinal cord and brainstem showed that remyelination restores rapid and therefore, probably, saltatory conduction $^{9}$. The spontaneous remyelination that occurs in cats following extensive demyelination caused by dietary manipulation leads to restoration of function, as measured by clinical examination ${ }^{23}$. However, whether remyelination leads to complete and sustained restoration of function will require more sophisticated analyses of neural circuit function and remains to be determined ${ }^{24}$, especially in situations in which a degree of axonal loss has 
already occurred. Given that remyelination leads to thinner myelin sheaths than myelination (see below), it is predicted from computational studies showing that velocities increase with myelin thickness that conduction will not completely return to normal ${ }^{25}$.

There may be other, longer-term effects of remyelination. The traditional view that the myelin sheath is fixed structurally after formation has been revised in light of studies showing that activation of phosphatidylinositol 3-kinase signalling, optogenetic stimulation of axonal activity or enrichment of the social environment can increase the thickness and/or number of sheaths ${ }^{26-28}$. Together, these results show that oligodendrocytes are able to respond to axonal and potentially other signals to alter sheath properties. This plasticity has been termed adaptive myelination and it raises the question as to whether this also provides a mechanism for learning, in which circuits that show sustained activity are reinforced by increased myelination. The fascinating question that follows on from these studies is whether the sheaths on remyelinated axons also show plasticity and, if not, does this limit any capacity for learning and thus contribute to the cognitive dysfunction seen in patients with MS?

An additional, similarly theoretical, concern over complete functional restoration comes from studies linking the formation of new oligodendrocytes to learning. Oligodendrocytes are born and generate new myelin sheaths throughout life $29-31$, and an important study showed that this new oligodendrocyte differentiation is required for motor learning in adult mice ${ }^{32}$. Although this work did not establish that myelination per se is required for motor learning the newly formed oligodendrocytes required for motor learning could have had other beneficial effects on axonal function, such as metabolic support - it did predict that any reduction in the number of progenitors in and around remyelinated lesions could have longerterm effects on learning by limiting this capacity for the generation of new oligodendrocytes. Although oligodendrocyte progenitor cell (OPC) numbers return to normal even after repeated acute episodes of demyelination-remyelination in rodents ${ }^{33-35}$, it is possible that exposure to a sustained demyelinating stimulus might lead to a depletion of OPC numbers ${ }^{36,37}$ and compromise this intriguing role of oligodendrocytes.

\section{[H1] Mechanisms of remyelination}

The keys stages in remyelination are now well established (FIG. 2). In response to demyelinating injury, adult progenitors undergo a change in state often referred to as activation, in which at least some of these cells in the vicinity of a lesion re-enter the cell cycle $^{38}$. This enables progenitors to populate and expand within areas of damage through a combination of proliferation and migration; finally, they undergo differentiation, a process culminating in the formation of new myelin sheaths ${ }^{39,40}$. These sheaths are often thinner than those formed during development, a characteristic widely used to distinguish areas of remyelination from normally myelinated axons ${ }^{41}$. Recent years have seen an explosion of studies identifying factors, both extrinsic (also described as environmental or non-cell autonomous) and intrinsic (cell autonomous) that are involved in each of these distinctive phases, of which the timely and seamless transition from one to next is essential for efficient remyelination ${ }^{42-47}$. Next, therefore, we will review some of these recent developments that 
together have transformed our understanding of the mechanisms of this important regenerative process and the reasons for its failure.

[H3] Adult oligodendrocyte progenitor cells. The developmental origin of oligodendrocytes was established over thirty years ago. They are derived from a now well-characterised population of progenitor cells whose name has gone through numerous iterations. Originally described as O-2A cells by Raff and colleagues in the 1980s (on account of their ability to generate a glial fibrillary acidic protein-expressing cell resembling an astrocyte in tissue culture, as well as oligodendrocytes) ${ }^{48}$, they have subsequently been called NG2 cells (based on their expression of a membrane bound protegogylcan), synantocytes, polydendrocytes, oligodendrocyte precursor cells and OPCs ${ }^{49-51}$. Similar cells that are derived from neonatal OPCs (nOPCs) and that persist into adulthood are called adult OPCs (aOPCs) ${ }^{52}$. These cells constitute approximately $6 \%$ of the CNS total cell number ${ }^{53}$ and are abundant throughout the CNS, where they generate new oligodendrocytes throughout life. They also receive glutamatergic and GABAergic synaptic inputs, and recent studies suggest that they have a potentially important role in modulating neuronal circuit activity ${ }^{54-57}$.

Given the central role of OPCs in developmental myelination, it seemed likely that aOPCs would be the cells that are responsible for generating new oligodendrocytes during the regenerative process of remyelination. Several lines of evidence strongly supported such a view, but it was not until the advent of genetic fate mapping strategies in which marker genes could be specifically expressed within aOPCs in such a way that their differentiation fates could be followed that the formal evidence that aOPCs are the major source of new oligodendrocytes could be confirmed ${ }^{39,40,58}$ (FIG. 2). More recent studies using dual-colour reporter mice that identify the developmental origin of aOPCs have revealed that those of dorsal developmental origin undergo enhanced recruitment and differentiation during remyelination compared with those of ventral origin, revealing a regenerative heterogeneity in aOPCs that is determined by developmental origin ${ }^{59}$.

Fate-mapping studies have also revealed alternative differentiation fates of aOPCs during tissue regeneration. Indeed, they have shown that aOPCs can generate astrocytes (albeit in small numbers compared with those generated from existing astrocytes) and, perhaps most surprisingly, Schwann cells that contribute to CNS remyelination in certain diseases and experimental models (BOX 2) ) $^{39,59}$. Thus, aOPCs are self-renewing multipotent cells; on this basis, a case can be made for regarding these cells as adult CNS stem cells ${ }^{60}$.

Although aOPCs constitute the overwhelmingly predominant source of new oligodendrocytes when one considers the entire CNS, progenitor populations within the sub-ventricular zone (SVZ) may also be able to generate new oligodendrocytes in an area of demyelination located near to the SVZ, such as the corpus callosum ${ }^{61-63}$. A long-standing question is whether preexisting mature oligodendrocytes might also be a source of new oligodendrocytes during remyelination. Genetic fate mapping shows that this is not the case ${ }^{64}$. However, pre-existing oligodendrocytes are able to increase the number of internodes they generate and thereby contribute to remyelination if extracellular signal-regulated kinases 1 and 2 (ERK1 and ERK2) 
are artificially activated 65 .

[H3] Activation of adult oligodendrocyte progenitor cells. Progenitor activation is the term used to describe the specific set of changes that occur in aOPCs in response to disruption of tissue homeostasis caused by injury. This process is closely associated with the proliferative response of aOPCs following tissue injury, but whether it occurs within aOPCs before they proliferate or is a feature of newly generated aOPCs is unclear. Early descriptions of activation described a change in aOPC morphology that was subsequently linked to the increased expression of several genes, many of which are transcription factors ${ }^{47,66,67}$. More recently, a thorough description of the changes in gene expression associated with activation have been acquired ${ }^{38}$. Through the use of reporter mice that enable fluorescence-activated cell sortingbased isolation of specific populations, it has been possible to generate gene expression profiles of aOPCs from intact white matter and from regions of demyelination, and compare these profiles with those of nOPCs and mature oligodendrocytes from adult CNS. This study revealed that the resting aOPC has an expression profile that more closely resembles a mature oligodendrocyte than an nOPC, but following demyelination, aOPCs in their activated state 'revert' to a transcriptome than more closely resembles their developmental forebears. This makes intuitive sense, as it is only nOPCs and activated aOPCs that need to prepare themselves for generating new oligodendrocytes, the former for myelination and the latter for remyelination.

The changes in gene expression associated with activation are clearly necessary for the ensuing regenerative process. Two examples provide evidence of the critical importance of this initial aOPC event. First, the transcription factor TCF7L2 (also known as TCF4) is only expressed in aOPCs following tissue injury (it is undetectable in oligodendrocyte lineage cells in normal adult $\mathrm{CNS})^{68}$. As discussed below, this transcription factor is at the heart of canonical WNT signalling and serves to maintain aOPCs in the cell cycle as their numbers increase to populate areas of demyelination during the recruitment phase of remyelination. The second example is provided by the transcription factor SOX2, which, like TCF7L2, is only expressed in aOPCs following tissue damage ${ }^{69}$. SOX2 appears to function as a master switch, with expression associated with an increase in aOPC proliferation and the priming these cells for eventual differentiation into myelinating oligodendrocytes.

The precise mechanisms for inducing activation are not known but they probably relate to the innate immune response that is triggered by tissue injury. A plausible working hypothesis involves the initial detection of the change in tissue integrity by microglia (presumably via pattern recognition receptors), the activation of these cells and the associated secretion of a battery of cytokines and other signalling molecules. These factors rapidly activate astrocytes, which secrete a range of factors leading to a rapid change in the signalling milieu of the tissue that is detected by aOPCs, causing their activation. It remains to be established how the recently identified heterogeneity in the response of astrocytes to CNS damage fits into this model of remyelination ${ }^{70}$. It is likely that signals emanating from the damaged tissue (such as damage-associated molecular patterns) also directly contribute to OPC activation. Although the process of remyelination is a regenerative sequela of primary demyelination, it seems that aOPC activation is not confined to this very specific 
form of pathology but occurs in all forms of CNS disturbance, and it is possible that aOPC activation might contribute to the resolution of other forms of CNS injury ${ }^{71}$ through other recently discovered biological functions of this cell population, such as the control of angiogenesis ${ }^{72}$.

[H3] Co-ordination of recruitment and differentiation. The next identifiable phase of remyelination is recruitment - the colonization of areas of demyelination with sufficient aOPCs to generate the number of oligodendrocytes required to restore myelination. Mirroring a common mechanism employed in development to regulate cell number, in which a surfeit of progenitors is generated and then subsequently pruned to the number of differentiated cells required, the initial progenitor response to demyelination is usually far in excess of that needed. This is invariably the case in experimental models, although in clinical disease the situation is less straightforward since there are, as we will discuss below, certainly instances where remyelination failure is associated with too few progenitors ${ }^{73,74}$. The recruitment of aOPCs to and within areas of demyelination depends to a large extent on cell division and also on cell migration, albeit this migration probably occurs over relatively short distances ${ }^{75}$.

An extensive literature now exists on the many factors than control both OPC division and migration and although only some of this literature relates to the study of aOPCs in the context of demyelination, it is likely than many of the mediators described in developmental and in vitro systems will contribute to the complex variety of factors regulating the recruitment phase of remyelination. The sources of both mitogens and regulators of migration are many and there are few, if any, constituents of a demyelinating lesion that do not contribute factors involved in aOPC recruitment. Cells of the innate immune system, be they microglia or recruited monocyte-derived macrophages, are a major source of factors that enhance aOPC activation, proliferation and migration. Astrocytes, activated by the acute injury, are a further source, as are cells of the vasculature and the aOPCs themselves ${ }^{38,76,77}$. The multiplicity of recruitment mediators raises the question of why so many factors are needed and from so many distinct sources? It seems likely that there are high levels of redundancy, with different factors mediating essentially the same function. However, it may also speak to a precisely choreographed sequence of events required for recruitment that we as yet have not fully understood.

A particularly important part of this choreography is the pathways that inhibit differentiation. These are closely linked to the control of the recruitment phase because preventing cells from exiting cell cycle by undergoing differentiation is an important part of establishing sufficient numbers of progenitors to ensure successful remyelination ${ }^{78}$. Two key pathways to have emerged as negative regulators of OPC differentiation are the Notch pathway, which in developmental myelination prevents differentiation ${ }^{79}$, and the canonical WNT pathway ${ }^{68}$. A clue to the importance of the WNT pathway was initially provided by the identification of the transcription factor TCF2L7 in oligodendrocyte lineage cells in remyelination ${ }^{68}$. This led to a series of studies that have not only yielded a detailed understanding of the WNT pathway in controlling the transition from the proliferation phase to the differentiation phase but also revealed insights into myelin pathology and opened up exciting possibilities by which 
remyelination might be therapeutically enhanced ${ }^{80-83}$.

[H3] Differentiation and the formation of thin myelin sheaths. The recruitment phase is followed by the differentiation phase, in which recruited aOPC extend processes around demyelinated axons and ultimately invest the axon with a new compacted myelin sheath. It is now well established in oligodendrocyte biology that there is an exclusivity between the mechanisms governing OPC proliferation and those that control the differentiation of OPCs into mature myelin-forming oligodendrocytes: for differentiation to occur, a cell must exit the cell cycle ${ }^{84}$, a transition in which SFMBT2 cluster miRNAs, MYC and E2F1 have key roles ${ }^{85-87}$. When remyelination proceeds smoothly, there is a timely transition from the recruitment to the differentiation phase. Dysregulation of the kinetics of this transition plays a large part in the declining efficiency of remyelination with age, as we discuss later. However, despite its importance, relatively little is known about how this transition occurs, although it clearly involves kinases that control cell cycle and cell cycle exit ${ }^{78}$. One possible mechanism relates to cell density, which, when it reaches a certain level, initiates differentiation (conversely, a decrease in density such as can occur during demyelination, is a stimulus to induce recruitment) ${ }^{88-90}$. The past few years have seen a considerable expansion in our understanding of the mechanisms of differentiation by which an aOPC transitions into a myelinating oligodendrocyte, including the identification of key transcription factors such as MRF and epigenetic regulators ${ }^{91-95}$. Not all of this information has been gleaned from models of demyelination and remyelination, but that which has is especially interesting as it opens up exciting opportunities for small molecule-based therapeutic interventions by which remyelination might be enhanced clinically (discussed below).

The last stage of oligodendrocyte differentiation is the formation of a new compacted myelin sheath. When myelination occurs during development there is a clear relationship between myelin sheath thickness (and length) and axon diameter. However, in remyelination, the new myelin sheath thickness and length show little increase with increasing axonal diameter. This means that, in remyelination, the myelin sheaths are thinner and shorter than the original sheaths generated during development ${ }^{41}$ (FIG. 3). Although some remodelling of the new myelin internode occurs, the original dimensions are only attained for small-diameter fibres ${ }^{96-}$ ${ }^{98}$. The relationship between axon diameter and myelin sheath thickness is expressed as the $g$ ratio, which is calculated as the fraction of the axon circumference to the axon plus myelin sheath circumference. The thin myelin sheaths characteristic of remyelination have a higher $g$ ratio than that of the normally myelinated axon and this remains the most reliable way of unambiguously identifying remyelination. However, while thin myelin sheaths are easily detected when large diameter axons are remyelinated, the situation is less straightforward for smaller-diameter axons such as those within the corpus callosum, where the normally thinner myelin sheaths mean that the $g$ ratios of remyelinated axons are often unchanged ${ }^{97}$.

An important question in myelin biology is how the relationship between the thickness and length of the myelin sheath and axon size is established in developmental myelination and why it should fail during remyelination? In the PNS, expression of neuregulin 1 (NRG1)-type III on axons is clearly important: less NRG1 results in thinner myelin sheath with an increased $g$ 
ratio, whereas more NRG1 results in an thicker myelin sheath with a decreased $g$ ratio ${ }^{99}$. In the CNS overexpression of NRG1 leads to hypermyelination in development. However, mice lacking expression of $\mathrm{Nrg} 1$ or both Erb3 and Erb4 (which encode NRG1 receptors) undergo normal myelination, indicating that NRG1 is not necessary for CNS myelination ${ }^{100}$ and other signals, including the cell-intrinsic mechanisms that enable oligodendrocytes to form sheaths on artificial fibres that mimic axons ${ }^{101}$, must contribute to the establishment of the myelin sheath parameters. However, none of the mechansistic insights into the control of myelin sheath formation in development shed light on the increased $g$ ratio in remyelination. For example, while increased expression of NRG1 increases myelin sheath thickness in development it fails to do so during remyelination ${ }^{100}$. Likewise, activation of the AKT pathway, a well-established determinant of myelin sheath thickness in development, does not result in thicker myelin sheaths in remyelination ${ }^{102}$. One hypothesis to explain the discrepancy between myelination and remyelination is that whereas oligodendrocytes myelinating during development associate with expanding axons that are still acquiring their final length and diameter and are able to induce adaptive changes in the myelin sheath, the remyelinating oligodendrocyte engages an axon that is comparatively static, having already acquired its final size. Thus, the remyelinating oligodendrocyte does not encounter the same dynamic stresses and other signals that might drive adaptive changes encountered by the myelinating oligodendrocyte ${ }^{103}$, and remyelination reflects largely the activity of the cell-intrinsic mechanisms (FIG. 3).

\section{[H1] Systemic factors and remyelination}

As with regenerative processes, remyelination is profoundly affected by systemic factors. Recent studies have emphasized two such factors: the essential role of the immune system and the profound impact of ageing on the process.

[H3] Remyelination and the immune system. Various lines of evidence strongly suggest that MS is primarily an autoimmune disease $\mathrm{e}^{104-106}$. However, the focus on the immunopathogenic nature of the maladaptive immune system in this disease has deflected attention from the role of the immune system and especially the innate immune system in remyelination. It is a well-established tenet of pathology that one of the functions of inflammation is to prepare damaged tissue for reparative processes, and it is now abundantly clear that the innate immune response to demyelination has important roles in remyelination. In non-immunemediated models of demyelination, this innate immune response is mediated by microglia and by monocytes recruited from the circulation. Both cell types have the capacity to develop into macrophages. Here, we use the term macrophage to refer to cells of both origins, unless a distinction is drawn between the two ${ }^{107}$.

A correlation between the abundance of debris-filled macrophages and the efficiency of remyelination was reported in early studies of remyelination following toxin-induced demyelination, in which the inflammatory response is the consequence of demyelination and not its cause, as in immune-mediated models of demyelination such as EAE. A causal relationship between the macrophage response and remyelination was demonstrated by the depletion of the circulating monocytes that give rise to a proportion of the lesion 
macrophages, which led to remyelination impairment ${ }^{108}$. Subsequent studies on the nature of the beneficial roles of macrophages focused on their ability to clear the myelin debris generated during demyelination by phagocytosis, or on the various factors they secrete that influence the behaviour of OPCs and their progeny ${ }^{109,110}$. Myelin contains inhibitors of OPC differentiation, which, in the intact CNS, are thought to prevent OPCS undergoing differentiation in the absence of an exposed axon, as to do so would probably lead to them undergoing apoptosis ${ }^{111-113}$. Myelin debris generated by demyelination and containing inhibitors of OPC differentiation therefore needs to be removed from the extracellular space so that it does not interfere with the final differentiation stage of remyelination ${ }^{114-117}$. This is the function of phagocytic macrophages and the efficiency with which they perform this task has a major influence on the efficiency of remyelination.

In addition to any phagocytic role, activated macrophages are a source of a wide spectrum of secreted signalling molecules that may stimulate remyelination directly or indirectly ${ }^{118}$. In recent years, many macrophage-derived molecules have been identified that have direct effects on OPCs (for example, CXCR4 $4^{119}$, tumour necrosis factor ${ }^{120}$, endothelin $2^{121}$ and activin- $A^{122}$ ), and it is likely that others will be identified. Macrophages may also have roles in extracellular matrix remodelling and in the metabolic support of axons and oligodendrocytes (via the release of lactate and iron, respectively) and the contribution of these roles to the regenerative function of macrophages will need to be clarified ${ }^{123}$. The precise nature of macrophage function is determined by the macrophage state, which is often referred to as being either 'classically activated' or M1, or 'alternatively activated' or M2. Although there are many caveats to this terminology, not least because it does not accurately reflect the multiple and interchangeable states that these cells can adopt, it nevertheless provides useful terms of convenience with which to identify distinctive macrophage contributions. The M1 state is prevalent during the recruitment phase of remyelination whereas the $M 2$ state is dominant and instrumental during the differentiation phase ${ }^{122}$. The timely transition from the $\mathrm{M} 1$ to the M2 state is critical for rapid and efficient remyelination. Although both resident microglia and recruited monocytes can both give rise to macrophages, it is becoming apparent that the two populations can have distinctive roles in CNS pathology ${ }^{124}$. Elucidating their distinctive roles, and that of the recently characterized non-parenchymal macrophages of the perivascular space and other brain borders ${ }^{125}$, will be necessary to fully understand the role of the innate immune system in remyelination.

The role of the adaptive immune response in remyelination has received relatively little attention. Early reports suggested a positive role for $\mathrm{T}$ cells in remyelination, as this process was impaired in their absence ${ }^{126,127}$. A more recent study identified a pro-remyelination role for regulatory T-cells present in MS lesions that is mediated in part by their expression of $\mathrm{CCN}^{128}$.

[H3] Ageing and remyelination. It is a common feature of regenerative processes that they become less efficient with ageing (which is one of the reasons why ageing occurs) ${ }^{129}$. Remyelination is no exception: it undergoes a progressive slowing in rate throughout adult life ${ }^{130-132}$, which may occur more rapidly in white matter than in grey matter, in which 
remyelination is thought to be more efficient ${ }^{133}$. As a result of the slowing of remyelination rate, demyelinated axons remain exposed for increasingly long periods ${ }^{134}$. As these axons depend on an intact myelin sheath for their survival, delays in remyelination leave axons increasingly vulnerable to degeneration ${ }^{22}$. Axonal loss is irreversible and as the number of lost axons accumulates, the degree of permanent clinical deterioration increases. Thus, the transition from treatable relapsing-remitting MS to untreatable chronic progressive MS probably occurs to a large extent on the age-associated decline in remyelination efficiency and the consequent degeneration of demyelinated axons.

Although it is difficult to know for certain at what rate remyelination occurs in people with MS, studies of patient cohorts support this hypothesis: individuals with MS reach specific levels of disability at around the same age regardless of the initial pattern of disease and the age of disease onset, pointing to an underlying age-associated decline in regenerative capacity ${ }^{135}$. Reports that remyelinated plaques can be found in long-lived individuals is not evidence against our hypothesis, as it is not possible to know at what age a lesion occurred or how long it took to remyelinate ${ }^{4}$. Rather, both imaging ${ }^{136}$ and pathology ${ }^{137}$ studies point to a strong age-effect on remyelination efficiency in MS.

There are several possible explanations for why remyelination efficiency declines with ageing. One possibility is that the density of aOPCs declines, leaving fewer cells available to be mobilized in response to demyelination. However, the available data indicate that there is no age-related decline in aOPC density ${ }^{138,139}$. However, there is evidence that aOPC activation, recruitment and differentiation are all impaired with increasing age ${ }^{138,139}$, and, of these, the effects on differentiation are especially rate limiting as increasing aOPC recruitment following experimental demyelination in aged mice does not lead to an acceleration in remyelination ${ }^{140}$. This emphasis on the failure of differentiation with ageing in animal models mirrors (and may well contribute to) the frequent occurrence of chronic demyelinating lesions containing oligodendrocyte lineage cells that have failed to undergo complete differentiation ${ }^{141-143}$

The ageing process affects both the intrinsic properties of aOPCs and the cells that form the extrinsic environment in which remyelination takes place. That intrinsic changes occur with ageing aOPCs is well-established, although the details of these changes have not been extensively explored, in part because of the technical challenges of growing aOPCs in tissue culture $^{144,145}$. The age-related changes in the remyelination environment are better understood, especially the contributions made by innate immune cells. There is not only an age-associated delay in the mobilization of the macrophage response but also a decrease in the ability of macrophages within lesions to clear myelin debris, which as described above contains factors that inhibit OPC differentiation $115,146,147$, and a delay in the switching of the macrophage population from one that is predominantly $\mathrm{M} 1$ to one that is predominantly $\mathrm{M} 2$, a switch that is important for the induction of OPC differentiation ${ }^{122}$.

An important question is whether ageing-associated effects are reversible. The answer to this question is critical in deciding whether to pursue regenerative therapies based on mobilizing 
the regenerative properties of endogenous stem and progenitor cell populations. A now widely used approach to address this question is the experimental procedure of heterochronic parabiosis, in which two adult mice of different ages are joined such that they share a common circulation ${ }^{148}$. This approach has been used to show that deficient remyelination in an aged mouse can be reversed, thus establishing the important principle that the effects of ageing on remyelination are reversible and validating therapeutic approaches based on targeting endogenous OPCs even in aged patients ${ }^{147}$. Such an approach is further validated by the enhancement of remyelination efficiency in aged rats using a small-molecule agonist of the nuclear hormone receptor retinoic acid receptor RXR ${ }^{149}$.

\section{[H1] Enhancing remyelination}

Clearly, the key first step in developing therapies that enhance remyelination and, in doing so, prevent neurodegeneration is the discovery of experimental strategies that promote or accelerate remyelination in relevant animal models. Two broad approaches have been taken: the identification of factors that normally inhibit remyelination (blockers of which will therefore promote the process) and the identification of those that accelerate the process.

[H3] Inhibitors of remyelination. A range of environmental components inhibit remyelination, including the extracellular matrix ${ }^{150-154}$; these components have been reviewed elsewhere ${ }^{155}$. Here, we focus on two signalling pathways that have received particular attention in view of their potential as targets for therapies to enhance remyelination. As discussed above, Notch signaling inhibits oligodendrocyte differentiation during development. The extent to which this pathway regulates OPC differentiation during remyelination is difficult to assess, despite the persistence of components of the pathway being implicated in remyelination failure in $\mathrm{MS}^{156}$. The expression of both Notch and Jagged in experimentally-induced areas of demyelination that undergo efficient remyelination, make it unlikely that their presence alone can account for remyelination block ${ }^{157}$. However, studies using an inducible Cre-lox approach to ablate Notch1 in OPCs following demyelination have yielded slightly different results depending on the type of Cre-driver used. In a study using a Plp1 promoter, there was no evidence in support of the prediction that ablation of Notch1 in progenitors caused premature progenitor differentiation and therefore accelerated remyelination, suggesting that Notch signalling is not a major regulator of OPC differentiation pathway during remyelination ${ }^{158}$. However, a subsequent study using the Olig1 promoter, which is expressed ay an earlier stage in oligodendrocyte development than Plp1, revealed an earlier onset of OPC differentiation, although this did not result in an overall increase in the rate of remyelination ${ }^{159}$. Thus, the canonical Notch pathway, whose activity in demyelinating lesions is enhanced by activated astrocyte-derived endothelin-1 ${ }^{76}$, seems to be one of the pathways that provides negative regulation of OPC differentiation, albeit not a dominant one. This may owe in part to competitive activation of non-canonical Notch signalling in OPCs that is involved in the induction of OPC differentiation ${ }^{160}$. An interesting take on the role of Notch signalling and differentiation has been provided by a careful examination of brain tissue from individuals with $\mathrm{MS}^{161}$ : since Notch-intracellular domain (NICD) is not present within the nucleus of OPCs present in chronically demyelinated lesions, it is unlikley to be able to activate downstream targets of the notch pathway. 
Leucine-rich repeat and immunoglobulin-like domain-containing nogo receptor-interacting protein 1 (LINGO-1) is a membrane-associated glycoprotein that is selectively expressed in the CNS. Originally shown to regulate axon outgrowth by interaction with the Nogo-66 receptor (NgR1) complex, it was subsequently also found to inhibit oligodendrocyte differentiation ${ }^{162}$. OPCs treated with small-interfering RNAs generated against LINGO-1, dominant negative LINGO-1 or LINGO-Fc led to cultured cells acquiring a more mature morphology. Consistent with this observation, LINGO-1 knock out mice exhibit precocious myelination in development ${ }^{162}$, whereas mice exposed to anti-LINGO-1 antibodies exhibit accelerated CNS remyelination in the lysolecithin model of demyelination-remyelination ${ }^{163}$. Thus, it appears that LINGO-1 signalling does play a role in controlling the differentiation of OPC during myelin regneration. However, whether this effect in the animal models is via expression of LINGO-1 on oligodendrocyte lineage cells, for which unambiguous evidence is sparse, or through its expression on axons ${ }^{164}$ is not clear.

Accelerators of remyelination. Recent years have seen the identification of several mechanisms by which OPCs in areas of demyelination can be induced to differentiate into myelin-sheath-forming oligodendrocytes. Perhaps the most novel of these is the discovery that demyelinated axons can be electrically active and form new glutamatergic synapses with OPCs present within areas of demyelination, which, through sensing axonal activity via AMPA and kainate receptors, cause OPCs to exit the cell cycle and undergo differentiation ${ }^{165-168}$.

OPC differentiation during remyelination can also be promoted through a class of heterodimeric nuclear receptors containing retinoid X-receptor (RXR)- $\gamma^{149}$. A role for RXR- $\gamma$ in remyelination was first identified during a transcriptomic screen of recruitment and differentiation stages of the remyelination of a well-established toxin-mediated model of demyelination, subsequent loss and gain of function studies both in vitro and in vivo revealed that receptor activation resulted in induction of progenitor differentiation. RXR- $\psi$ is promiscuous in its choice of binding partner and several of these partners, such as thyroid hormone receptor and peroxisome proliferator-activated receptor- $\gamma$, are well-recognised regulators of OPC differentiation, whereas others such as liver $X$ receptor that also regulate myelination have less well-characterized roles in the process ${ }^{169,170}$. Recently, vitamin D receptor was identified as a key RXR- $\gamma$-binding partner in the control of OPC differentiation ${ }^{171}$, revealing a possible role for vitamin $D$ in the regenerative component of demyelinating disease, in addition to its well-documented role as a susceptibility factor for $\mathrm{MS}^{172}$. Given the multiple potential binding partners of RXR- $\psi$, an as yet unproven model has emerged in which RXR- $\gamma$ switches its principal binding partner as OPCs proceed through distinctive stages of progression from dividing cells, to cells that exits cell cycle, initiate differentiation and ultimately become myelinating or remyelinating cells.

\section{[H3] Identifying remyelination drugs}

Given our expanding knowledge of the mechanisms of remyelination and the evidence for its neuroprotective and functional effects, the development of drug-based therapies for enhancing remyelination in MS and other myelin diseases is now a priority for academia and 
pharma alike. Two broad approaches have been taken to the discovery of small molecule or biological leads that target these stages (FIG. 4).

The first of these approaches has been the targeting of specific intrinsic or extrinsic signals that regulate the different stages of remyelination. This approach has led to the identification of a plethora of potential drugs and targets ${ }^{150,173-187}$ and to the first human trials of drugs designed specifically to enhance remyelination in MS. Humanized monoclonal antibodies against LINGO showed promise in early trials in optic neuritis ${ }^{188}$ but failed to meet primary endpoints in a Phase 2 trial in MS (ClinicalTrials.gov Identifier: NCT01864148). Other candidates within these regulatory signals are being explored as targets in pre-clinical studies, but here the lack of a single animal model that recapitulates the features of progressive MS (as discussed in BOX 1 ) is a significant impediment. Moreover, it is only when using toxin models in aged animals that one can generate lesions that remyelinate so slowly that they mimic remyelination failure in MS and also better resemble the age of patients most in need of and most likely to benefit from regenerative therapies ${ }^{149}$. An optimal approach will therefore require a combination of models for pre-clinical development of small molecules or biologics. Even then, this lack of a single model increases the risk that apparently promising leads will fail in clinical trials, in part because studies in such models will fail to address important interactions between the inflammatory, regenerative and neurodegenerative processes. For example, the use of separate inflammatory and regenerative models makes it more difficult to assess the balance between the benefits of profound suppression of disease by aggressive immunoablative therapies such as humanised monoclonal antibodies or bone marrow transplantation and the risks of losing the alternatively-activated microglial cell populations described above and the potential impact on the regenerative response.

A key question for the selection of suitable candidates for pre-clinical work is at what point does the remyelination process fail during attempted regeneration in MS? Clearly, a drug designed to promote a stage of remyelination that is already occurring efficiently during the regenerative process will be less effective than one that targets a blocked stage directly. However, it is clear from neuropathological studies that MS lesions are heterogeneous. Influential studies over the past two decades have defined different patterns of inflammation within MS lesions ${ }^{189}$, and more recent studies examining the regenerative response reveal further heterogeneity, in that $30 \%$ of lesions lack sufficient OPCs for remyelination whereas in the remainder, sufficient OPCs are present but remyelination fails at the later stages of differentiation and/or myelin sheath formation ${ }^{73,190}$. These studies show immediately that treatments targeting oligodendrocyte differentiation would only be effective in $70 \%$ of lesions, with the remainder requiring treatments that promote progenitor activation and migration. If these $70 \%$ then have further heterogeneity in terms of the stage at which the process of remyelination is blocked, then drugs targeting only one stage of the process will be even less effective and combination therapies targeting each specific blocked stage will be required. It follows that detailed neuropathological studies of the regenerative process are required, with the application of technologies such as single cell RNAseq on post mortem human material to better define the cell types and stages of differentiation within lesions when informative antibodies are not available. 
The second approach to remyelination-promoting drugs is the use of unbiased high-content screens examining oligodendrocyte behaviour in response to libraries of small molecules or FDA-approved drugs, with the regenerative activity of the compounds showing positive effects confirmed by assays of remyelination ${ }^{191-194}$. A number of such screens have been performed, using either primary cells or pluripotent stem cell-derived oligodendrocyte progenitors (FIG. 4). All bar one of these studies examined oligodendrocyte differentiation as an endpoint, as measured by the expression of myelin proteins. The one that did not used an ingenious micropillar design to examine the next stage of oligodendrocyte differentiation, the formation of sheets of membrane that wrap around 3D shapes - in this case the micropillar cones - and thus examined the first steps of myelin sheath formation ${ }^{191}$. Each of the studies has identified compounds that enhance differentiation. For some compounds, such as the FDA-approved drugs miconazole and clobetasol, there were no obvious signalling pathways responsible, although they appear to activate mitogen-activated protein kinase and glucocorticoid receptor signalling, respectively. For others, however, such as the antimuscarinic drugs benzatropine and clemastine, novel pathways regulating oligodendrocyte differentiation have been identified and confirmed in experimental studies ${ }^{193,195}$.

As these hits are FDA-approved drugs, the progression to clinical trials is facilitated, and one trial using clemastine has already been completed (ClinicalTrials.gov Identifier:

NCT02040298), although the outcome has not been reported yet. The analysis of this and other trials will be an important landmark and show clearly that the field has progressed into the area of experimental medicine. Thus far, however, the screens used to test FDA-approved and other libraries have been predicated on the assumption that oligodendrocyte differentiation and/or wrapping is a rate-limiting step for remyelination in MS lesions, and that sheath formation and reconstruction of the nodes of Ranvier will follow. Given the highly complex structure of the multilamelar sheath and the node, and the evidence already available from cell biology studies that reveal novel roles for cytoskeletal actin depolymerisation and polarity proteins in sheath formation ${ }^{46,196}$, this assumption may not be justified. Further screens focused on these later stages of remyelination, and on steps prior to oligodendrocyte differentiation identified in the neuropathology studies as possible points of arrest in the remyelination process, may be required.

\section{[H3] Cell therapies}

An alternative approach to remyelination, but one that is logically appropriate only in those lesions in which OPCs are not present, is cell replacement by transplantation. Compelling experimental evidence that cell transplantation may restore myelination first came in the 1980s. Patches of myelination were observed following transplantation of wild-type cells into shiverer mutant mice ${ }^{197}$, which lack normal compacted myelin as a result of a deletion in the myelin basic protein gene. Subsequently, transplantation of myelin-forming cells into focal demyelinated lesions generated by toxin injection was shown to result in remyelination ${ }^{198,199}$. More recent work has shown that such restoration can be extensive as transplanted rodent or human cells can myelinate the entire CNS of shiverer mice $e^{200-202}$.

When considered in the context of MS, however, the problems of transplantation into multiple lesions each with a chronic inflammatory and potentially adverse environment 
become germane. A much easier challenge for cell transplantation would be the hypomyelinating leucodystrophies - genetic diseases in which oligodendrocytes fail to form normal myelin - and here the spectacular results of the shiverer mice transplantation experiments are more clearly relevant to the clinical situation. A transplantation trial using human CNS stem cells that have the ability to differentiate into oligodendrocytes has been performed in children with a severe conatal form of one of these leucodystrophies, PelizaeusMerzbacher disease, caused by a mutation in $P L P 1^{203}$. Although no adverse effects were reported in the four children, MRI suggested that only of a modest degree of myelination had occurred near to the injection site. Two factors may have contributed to the degree of myelination observed compared with that seen in the rodent studies. First, the degree of migration of the transplanted cells may be limited, with the major differences in size between the rodent and human brain therefore becoming a limiting factor. Second, the cell populations used in the clinical trial were, inevitably given the need to perform prolonged testing so as to generate good manufacturing practices-grade cells and a satisfactory safety profile, generated using protocols no longer regarded as state-of-the-art by stem cell biologists interested in creating oligodendrocytes. They were therefore likely less efficient at generating myelin-forming oligodendrocytes than the primary fetal or pluripotent based populations used in the shiverer mice studies.

This trial illustrates the scale of the challenge for cell therapies to promote remyelination, and it seems premature to consider transplantation in MS without first establishing efficacy in the much more propitious environment of the developing brain. For this, the numbers of patients suitable for transplantation will probably be small and it will be important to consider other conditions in which myelination is prevented owing to oligodendrocyte defects, such as radiation-induced or chemotherapy-induced oligodendrocyte progenitor depletion and white-matter damage in children being treated for tumours ${ }^{204,205}$. An additional challenge is provided by cell availability. The use of primary fetal cells will be extremely limited owing to their availability, making anything more than proof-of-principle studies difficult. Induced pluripotent stem cells differentiated into oligodendrocytes and their progenitors provide an attractive alternative as this would overcome the need for immunosuppression, but their safety remains unproven and many lines generate tumours post transplantation ${ }^{206}$. Embryonic stem (ES) cell-derived oligodendrocytes will probably be the cell of choice, with robust differentiation protocols in place ${ }^{207}$ and with an ongoing clinical trial using ES cellderived retinal pigment epithelial cells providing the important proof of principle that ES-cell based therapies will meet regulatory standards of safety.

\section{[H3] The future - experimental medicines}

Key milestones for the field will be the early phase clinical trials that demonstrate efficacy of a drug or a cell in promoting remyelination. These will, by enabling subsequent cell-based and animal-based studies to be designed around questions raised from studies of the trial participants, herald the arrival of a genuinely iterative experimental medicine approach to remyelination. There are, however, as discussed in BOX 3 , major challenges for these trials in the development of outcome measures that are sufficiently sensitive to detect the regenerative effects of the drug under trial and, equally importantly, ensure that a positive effect is not missed. Overcoming these challenges will require the further development of 
biomarkers for regeneration, and this must now be a major goal for the field. Once these are in place we predict that this 'bench-to-bedside-to-bench-again' approach will lead to genuinely effective regenerative therapies that complement the immunomodulatory drugs developed over the past two decades for MS and thus provide effective treatments for progressive MS. 


\section{Box 1 | Experimental models of remyelination}

Experimental models can be 'disease models' that provide as close a facsimile of the naturally occurring disease, or they can 'mechanisms models' that are more reductionist, allowing focused analysis of a specific aspect of a complex pathology. Experimental autoimmune encephalomyelitis (EAE), in its many guises, is commonly thought to provide a disease model of multiple sclerosis (MS). This is however incorrect - although EAE can be induced in a focal manner ${ }^{131,208}$, mimicking an acute MS lesion, and can develop into a chronic inflammatory state in some rodent genetic backgrounds, it does not recreate the combination of acute and chronic inflammation, regeneration and neurodegeneration that characterizes progressive MS. There are in fact no disease models for MS, and it is more correct to think of EAE as a mechanisms model for the immunopathogenesis of MS, and not as a model that lends itself to the study of the neurobiological aspects of the disease, including remyelination. Instead, this requires other mechanisms models. These generally involve the use of toxins that kill oligodendrocytes (hence leading to primary demyelination, the substrate for remyelination) and, to varying degrees, other cells types. The models commonly in use involve i) injection of lysolecithin into the spinal cord or corpus callosum white matter in mice or rats, ii) injection of ethidium bromide into cerebellar peduncles in rats or into the spinal cord in rats or mice, or iii) oral administration of cuprizone in mice. In each of these models (albeit to a lesser extent in the cuprizone model), the site of demyelination is anatomically defined and the process of demyelination is temporally separated from the subsequent process of remyelination, allowing the latter to be specifically studied without the complication of ongoing demyelination. These mechanisms models therefore allow the fundamental biology of remyelination to be elucidated without the confounding and complicating involvement of an autoimmune process. They, like EAE, do not provide a facsimile of MS. Nevertheless, they are of great value as the fundamental mechanisms of remyelination will be applicable to all forms of demyelination regardless of how it is induced, be it toxin or immune mediated. This is consistent with a general concept in regenerative biology that the mechanism of regeneration is independent of the mechanism by which injury occurs. 


\section{Box 2 | Schwann cell remyelination in the CNS}

CNS remyelination can sometimes be mediated by Schwann cells as well as by oligodendrocytes. This unusual phenomenon occurs in a number of pathological conditions including multiple sclerosis, genetic disorders of myelination and traumatic spinal cord injury, and can be reproduced in a variety of in vivo experimental models. For many years it was assumed that Schwann cells remyelinating CNS axons were derived from PNS sources, and that they responded to recruitment signals generated by demyelination and migrated from these PNS sources into the CNS. This seemed a very plausible explanation given, first, the frequent anatomical distribution of CNS Schwann cells, often close to likely PNS sources such as spinal roots, and, second, that CNS Schwann cells occur in CNS regions that are deficient in astrocytes, suggesting that a breach in the astrocytic glia limitans of the CNS presents an opportunity for peripherally derived Schwann cells to 'flood' into CNS territories. However, genetic fate-mapping studies have revealed that although some CNS remyelinating Schwann cells are of PNS origin, the majority are derived from CNS progenitors. Several key questions remain regarding the phenomenon of CNS progenitor-mediated Schwann cell remyelination of the CNS. First, are CNS-derived Schwann cells the same as neural crest-derived Schwann cells of the PNS? Second, how do adult CNS progenitors become Schwann cells? Third, does it make any difference if a CNS axon is myelinated by an oligodendrocyte or a Schwann cell? Fourth, if it makes no difference, would strategies to prevent or promote remyelination by oligodendrocytes or Schwann cells be of therapeutic importance? 


\section{Box 3 | Designing clinical trials and developing outcome measures}

The trial design required to demonstrate the efficacy for any regenerative therapy in multiple sclerosis (MS) is complicated by the difficult question of outcome measures. Unlike tissues such as skin and liver, the brain cannot be tested by biopsy, so indirect measures of the efficacy of regenerative medicines are required. For remyelination, these measures currently comprise clinical assessment, imaging and electrophysiology, with each having its disadvantages. The clinical phenotype reflects a combination of inflammation, neurodegeneration and regeneration, so it is relatively insensitive to remyelination alone. MRI has revolutionised our ability to detect inflammatory lesions in MS, but imaging remyelination remains challenging $203,209,210$. Experimental and correlative neuropathological studies have suggested that the magnetisation transfer ratio (MTR) is sensitive to remyelination, and the wider availability of 7T scanners may also improve our ability to detect regeneration ${ }^{211-213}$. Another imaging strategy, positron emission tomography (PET) to detect a radiolabelled compound that incorporates into myelin, may also provide an approach to quantifying remyelination, with promising results from a study in MS patients in which enhanced signals within lesions correlates with a reduction in disability ${ }^{214,215}$. Further studies of all three are in progress. Electrophysiological techniques such as visual evoked potentials to measure conduction velocities represents a logical strategy to show remyelination as this would detect the reappearance of fast conduction velocities that are predicted to follow the restoration of salutatory conduction ${ }^{188,216,217}$. However, the sensitivity and specificity of this technique, in which the degree of variation can be considerable, remains to be determined. Also, the experimental data from spinal cord showing that the myelin sheaths formed by new (remyelinating) oligodendrocytes do not reach normal lengths until months after their formation raises the possibility that conduction velocities increase equally slowly and that trial protocols need to be prolonged appropriately.

These concerns over outcome measures are important when one considers that the likely effect size in early trials will be small, and are amplified by the issue of lesion heterogeneity (see main text). There are at present no clinical investigations that will distinguish lesions containing or lacking sufficient oligodendrocytes for myelination. Without the ability to separate these lesions, the power of trials for regenerative medicines targeted either at promoting progenitor migration or oligodendrocyte differentiation will be greatly diminished by the confounding effect of patients within the trial groups for whom the treatment under examination would never have any beneficial effect. The danger is therefore that a genuinely positive result that could guide further experimental work will be missed not because it did not work but because the effect could not be detected, resulting in a treatment strategy being abandoned permanently and prematurely. There is therefore an urgent need for strategies, most likely in our view to be PET, that enable reliable quantification of lesion heterogeneity and remyelination within and between individuals with MS. With such a technology both a rational stratification of patient cohorts and an accurate measurement of effect could be achieved, allowing selection of those patients most appropriate for any specific experimental medicine trial of a regenerative therapy and confident detection of any benefit. 
1. Franklin, R. J. M. Why does remyelination fail in multiple sclerosis? Nat. Rev. Neurosci. 3, 705-714 (2002).

2. Franklin, R. J. M. \& ffrench-Constant, C. Remyelination in the CNS: from biology to therapy. Nat. Rev. Neurosci. 9, 839-855 (2008).

3. Prineas, J. W., Barnard, R. O., Kwon, E. E., Sharer, L. R. \& Cho, E. S. Multiple sclerosis: remyelination of nascent lesions. Ann. Neurol. 33, 137-151 (1993).

This paper showed by electron microscopy that remyelination could be observed in MS lesions - a critical observation that underpins current research strategies to identify drugs and/or cells that enhance the process

4. Patrikios, P. et al. Remyelination is extensive in a subset of multiple sclerosis patients. Brain 129, 3165-3172 (2006).

5. Patani, R., Balaratnam, M., Vora, A. \& Reynolds, R. Remyelination can be extensive in multiple sclerosis despite a long disease course. Neuropathol. Appl. Neurobiol. 33, 277-287 (2007).

6. Nave, K.-A. Myelination and the trophic support of long axons. Nat. Rev. Neurosci. 11, 275283 (2010).

7. Franklin, R. J. M., ffrench-Constant, C., Edgar, J. M. \& Smith, K. J. Neuroprotection and repair in multiple sclerosis. Nat Rev Neurol 8, 624-634 (2012).

8. Frühbeis, C. et al. Neurotransmitter-triggered transfer of exosomes mediates oligodendrocyte-neuron communication. PLoS Biol. 11, e1001604 (2013).

9. Smith, K. J., Blakemore, W. F. \& McDonald, W. I. Central remyelination restores secure conduction. Nature 280, 395-396 (1979).

This seminal paper in remyelination biology established that remyelination can restore efficient impulse conduction to demyelinated axons.

10. Lee, Y. et al. Oligodendroglia metabolically support axons and contribute to neurodegeneration. Nature 487, 443-448 (2012).

11. Fünfschilling, U. et al. Glycolytic oligodendrocytes maintain myelin and long-term axonal integrity. Nature 485, 517-521 (2012).

12. Morrison, B. M., Lee, Y. \& Rothstein, J. D. Oligodendroglia: metabolic supporters of axons. Trends Cell Biol. 23, 644-651 (2013).

13. Arancibia-Carcamo, I. L. \& Attwell, D. The node of Ranvier in CNS pathology. Acta Neuropathol. 128, 161-175 (2014).

14. Rasband, M. N. \& Peles, E. The Nodes of Ranvier: Molecular Assembly and Maintenance. Cold Spring Harb Perspect Biol 8, a020495 (2015).

15. Dutta, R. \& Trapp, B. D. Mechanisms of neuronal dysfunction and degeneration in multiple sclerosis. Prog. Neurobiol. 93, 1-12 (2011).

16. Kornek, B. et al. Multiple sclerosis and chronic autoimmune encephalomyelitis: a comparative quantitative study of axonal injury in active, inactive, and remyelinated lesions. AJPA 157, 267-276 (2000).

17. Witte, M. E., Mahad, D. J., Lassmann, H. \& van Horssen, J. Mitochondrial dysfunction contributes to neurodegeneration in multiple sclerosis. Trends Mol Med 20, 179-187 (2014).

18. Dutta, R. et al. Mitochondrial dysfunction as a cause of axonal degeneration in multiple sclerosis patients. Ann. Neurol. 59, 478-489 (2006).

19. Lindner, M., Fokuhl, J., Linsmeier, F., Trebst, C. \& Stangel, M. Chronic toxic demyelination in the central nervous system leads to axonal damage despite remyelination. Neurosci. Lett. 453, 120-125 (2009).

20. Irvine, K. A. \& Blakemore, W. F. Remyelination protects axons from demyelinationassociated axon degeneration. Brain 131, 1464-1477 (2008).

21. Pohl, H. B. F. et al. Genetically induced adult oligodendrocyte cell death is associated with poor myelin clearance, reduced remyelination, and axonal damage. J. Neurosci. 31, 10691080 (2011).

22. Mei, F. et al. Accelerated remyelination during inflammatory demyelination prevents axonal loss and improves functional recovery. Elife 5, 1174 (2016).

In this paper the authors provide experimental evidence for the axon-protective benefits of 
remyelination and hence the likely therapeutic benefits of remyelination-enhancing treatments.

23. Duncan, I. D., Brower, A., Kondo, Y., Curlee, J. F. \& Schultz, R. D. Extensive remyelination of the CNS leads to functional recovery. Proc. Natl. Acad. Sci. U.S.A. 106, 6832-6836 (2009).

This paper uses an unusual dietary model of demyelination in cats to provide convincing evidence that remyelination of extensive areas of demyelination leads to functional recovery and reversal of clinical signs.

24. Manrique-Hoyos, N. et al. Late motor decline after accomplished remyelination: impact for progressive multiple sclerosis. Ann. Neurol. 71, 227-244 (2012).

25. Smith, R. S. \& Koles, Z. J. Myelinated nerve fibers: computed effect of myelin thickness on conduction velocity. Am. J. Physiol. 219, 1256-1258 (1970).

26. Flores, A. I. et al. Constitutively active Akt induces enhanced myelination in the CNS. J. Neurosci. 28, 7174-7183 (2008).

27. Gibson, E. M. et al. Neuronal Activity Promotes Oligodendrogenesis and Adaptive Myelination in the Mammalian Brain. Science 1252304 (2014). doi:10.1126/science.1252304

28. Liu, J. et al. Impaired adult myelination in the prefrontal cortex of socially isolated mice. Nat. Neurosci. 15, 1621-1623 (2012).

29. Young, K. M. et al. Oligodendrocyte dynamics in the healthy adult CNS: evidence for myelin remodeling. Neuron 77, 873-885 (2013).

30. Rivers, L. E. et al. PDGFRA/NG2 glia generate myelinating oligodendrocytes and piriform projection neurons in adult mice. Nat. Neurosci. 11, 1392-1401 (2008).

31. Horner, P. J. et al. Proliferation and differentiation of progenitor cells throughout the intact adult rat spinal cord. J. Neurosci. 20, 2218-2228 (2000).

32. McKenzie, I. A. et al. Motor skill learning requires active central myelination. Science 346, 318-322 (2014).

33. Penderis, J., Shields, S. A. \& Franklin, R. J. M. Impaired remyelination and depletion of oligodendrocyte progenitors does not occur following repeated episodes of focal demyelination in the rat central nervous system. Brain 126, 1382-1391 (2003).

34. Rodriguez, E. G. et al. Oligodendroglia in cortical multiple sclerosis lesions decrease with disease progression, but regenerate after repeated experimental demyelination. Acta Neuropathol. 128, 231-246 (2014).

35. Yeung, M. S. Y. et al. Dynamics of oligodendrocyte generation and myelination in the human brain. Cell 159, 766-774 (2014).

36. Mason, J. L. et al. Oligodendrocytes and progenitors become progressively depleted within chronically demyelinated lesions. Am. J. Path. 164, 1673-1682 (2004).

37. Vana, A. C. et al. Platelet-derived growth factor promotes repair of chronically demyelinated white matter. J. Neuropathol. Exp. Neurol. 66, 975-988 (2007).

38. Moyon, S. et al. Demyelination causes adult CNS progenitors to revert to an immature state and express immune cues that support their migration. J. Neurosci. 35, 4-20 (2015).

This paper provides the first comprehensive description of the process by which resting adult CNS progenitors become activated, priming them for the ensuing regenerative process of remyelination.

39. Zawadzka, M. et al. CNS-resident glial progenitor/stem cells produce Schwann cells as well as oligodendrocytes during repair of CNS demyelination. Cell Stem Cell 6, 578-590 (2010).

40. Tripathi, R. B., Rivers, L. E., Young, K. M., Jamen, F. \& Richardson, W. D. NG2 glia generate new oligodendrocytes but few astrocytes in a murine experimental autoimmune encephalomyelitis model of demyelinating disease. J. Neurosci. 30, 16383-16390 (2010).

41. Blakemore, W. F. Pattern of remyelination in the CNS. Nature 249, 577-578 (1974).

42. Swiss, V. A. et al. Identification of a gene regulatory network necessary for the initiation of oligodendrocyte differentiation. PLOS ONE 6, e18088 (2011).

43. Gaesser, J. M. \& Fyffe-Maricich, S. L. Intracellular signaling pathway regulation of myelination and remyelination in the CNS. Exp. Neurol. 283, 501-511 (2016).

44. Steelman, A. J. et al. Activation of oligodendroglial Stat3 is required for efficient remyelination. Neurobiol. Dis. 91, 336-346 (2016).

45. He, D. et al. Chd7 cooperates with Sox10 and regulates the onset of CNS myelination and remyelination. Nat. Neurosci. 19, 678-689 (2016).

46. Jarjour, A. A. et al. The polarity protein Scribble regulates myelination and remyelination in the central nervous system. PLoS Biol. 13, e1002107 (2015).

47. Nakatani, H. et al. Ascl1/Mash1 promotes brain oligodendrogenesis during myelination and 
remyelination. J. Neurosci. 33, 9752-9768 (2013).

48. Raff, M. C., Miller, R. H. \& Noble, M. A glial progenitor cell that develops in vitro into an astrocyte or an oligodendrocyte depending on culture medium. Nature 303, 390-396 (1983).

49. Richardson, W. D., Young, K. M., Tripathi, R. B. \& McKenzie, I. NG2-glia as multipotent neural stem cells: fact or fantasy? Neuron 70, 661-673 (2011).

50. Butt, A. M., Kiff, J., Hubbard, P. \& Berry, M. Synantocytes: new functions for novel NG2 expressing glia. J. Neurocytol. 31, 551-565 (2002).

51. Nishiyama, A., Komitova, M., Suzuki, R. \& Zhu, X. Polydendrocytes (NG2 cells): multifunctional cells with lineage plasticity. Nat. Rev. Neurosci. 10, 9-22 (2009).

52. ffrench-Constant, C. \& Raff, M. C. Proliferating bipotential glial progenitor cells in adult rat optic nerve. Nature 319, 499-502 (1986).

The first evidence for adult OPCs (aOPCs) in the CNS. These cells generate the new oligodendrocytes that remyelinate axons.

53. Dawson, M. R. L., Polito, A., Levine, J. M. \& Reynolds, R. NG2-expressing glial progenitor cells: an abundant and widespread population of cycling cells in the adult rat CNS. Mol. Cell. Neurosci. 24, 476-488 (2003).

54. Bergles, D. E., Roberts, J. D., Somogyi, P. \& Jahr, C. E. Glutamatergic synapses on oligodendrocyte precursor cells in the hippocampus. Nature 405, 187-191 (2000).

55. Sakry, D. et al. Oligodendrocyte precursor cells modulate the neuronal network by activitydependent ectodomain cleavage of glial NG2. PLoS Biol. 12, e1001993 (2014).

56. Birey, F. et al. Genetic and Stress-Induced Loss of NG2 Glia Triggers Emergence of Depressive-like Behaviors through Reduced Secretion of FGF2. Neuron 88, 941-956 (2015).

57. Lin, S.-C. \& Bergles, D. E. Synaptic signaling between GABAergic interneurons and oligodendrocyte precursor cells in the hippocampus. Nat. Neurosci. 7, 24-32 (2004).

58. Hesp, Z. C. et al. Chronic oligodendrogenesis and remyelination after spinal cord injury in mice and rats. J. Neurosci. 35, 1274-1290 (2015).

59. Crawford, A. H., Tripathi, R. B., Richardson, W. D. \& Franklin, R. J. M. Developmental Origin of Oligodendrocyte Lineage Cells Determines Response to Demyelination and Susceptibility to Age-Associated Functional Decline. Cell Rep 15, 761-773 (2016).

60. Crawford, A. H., Stockley, J. H., Tripathi, R. B., Richardson, W. D. \& Franklin, R. J. M. Oligodendrocyte progenitors: Adult stem cells of the central nervous system? Exp. Neurol. 260, 50-55 (2014).

61. Xing, Y. L. et al. Adult neural precursor cells from the subventricular zone contribute significantly to oligodendrocyte regeneration and remyelination. J. Neurosci. 34, 1412814146 (2014).

62. Samanta, J. et al. Inhibition of Gli1 mobilizes endogenous neural stem cells for remyelination. Nature 526, 448-452 (2015).

63. Kazanis, I. et al. Subependymal Zone-Derived Oligodendroblasts Respond to Focal Demyelination but Fail to Generate Myelin in Young and Aged Mice. Stem Cell Reports (2017). doi:10.1016/j.stemcr.2017.01.007

64. Crawford, A. H. et al. Pre-Existing Mature Oligodendrocytes Do Not Contribute to Remyelination following Toxin-Induced Spinal Cord Demyelination. Am. J. Pathol. 186, 511516 (2016).

65. Jeffries, M. A. et al. ERK1/2 Activation in Preexisting Oligodendrocytes of Adult Mice Drives New Myelin Synthesis and Enhanced CNS Function. J. Neurosci. 36, 9186-9200 (2016).

66. Levine, J. M. \& Reynolds, R. Activation and proliferation of endogenous oligodendrocyte precursor cells during ethidium bromide-induced demyelination. Exp. Neurol. 160, 333-347 (1999).

67. Fancy, S. P. J., Zhao, C. \& Franklin, R. J. M. Increased expression of Nkx2.2 and Olig2 identifies reactive oligodendrocyte progenitor cells responding to demyelination in the adult CNS. Mol. Cell. Neurosci. 27, 247-254 (2004).

68. Fancy, S. P. J. et al. Dysregulation of the Wnt pathway inhibits timely myelination and remyelination in the mammalian CNS. Genes Dev. 23, 1571-1585 (2009).

By screening transcription factors expressed during remyelination the authors were able to identify the wnt signaling pathway as a potent negative regulator of progenitor differentiation during myelination and remyelination and spawning a series of further studies. 
69. Zhao, C. et al. Sox2 Sustains Recruitment of Oligodendrocyte Progenitor Cells following CNS Demyelination and Primes Them for Differentiation during Remyelination. J. Neurosci. 35, 11482-11499 (2015).

70. Liddelow, S. A. et al. Neurotoxic reactive astrocytes are induced by activated microglia. Nature 541, 481-487 (2017).

71. McTigue, D. M., Wei, P. \& Stokes, B. T. Proliferation of NG2-positive cells and altered oligodendrocyte numbers in the contused rat spinal cord. J. Neurosci. 21, 3392-3400 (2001).

72. Yuen, T. J. et al. Oligodendrocyte-encoded HIF function couples postnatal myelination and white matter angiogenesis. Cell 158, 383-396 (2014).

73. Boyd, A., Zhang, H. \& Williams, A. Insufficient OPC migration into demyelinated lesions is a cause of poor remyelination in MS and mouse models. Acta Neuropathol. 125, 841-859 (2013).

This paper shows that some MS lesions lack sufficient aOPCs to drive remyelination. This evidence for lesion heterogeneity is important, as it emphasizes that different lesions may require different treatment strategies to enhance remyelination - in some promoting differentiation may suffice whilst in others increasing aOPC numbers will be required.

74. Wegener, A. et al. Gain of Olig2 function in oligodendrocyte progenitors promotes remyelination. Brain 138, 120-135 (2015).

75. Franklin, R. J. M., Gilson, J. M. \& Blakemore, W. F. Local recruitment of remyelinating cells in the repair of demyelination in the central nervous system. J. Neurosci. Res. 50, 337-344 (1997).

76. Hammond, T. R. et al. Astrocyte-derived endothelin-1 inhibits remyelination through notch activation. Neuron 81, 588-602 (2014).

77. Arai, K. \& Lo, E. H. An oligovascular niche: cerebral endothelial cells promote the survival and proliferation of oligodendrocyte precursor cells. J. Neurosci. 29, 4351-4355 (2009).

78. Caillava, C. et al. Cdk2 loss accelerates precursor differentiation and remyelination in the adult central nervous system. J. Cell Biol. 193, 397-407 (2011).

79. Wang, S. et al. Notch receptor activation inhibits oligodendrocyte differentiation. Neuron 21, 63-75 (1998).

80. Fancy, S. P. J. et al. Axin2 as regulatory and therapeutic target in newborn brain injury and remyelination. Nat. Neurosci. 14, 1009-1016 (2011).

81. Lee, H. K. et al. Apcdd1 stimulates oligodendrocyte differentiation after white matter injury. Glia 63, 1840-1849 (2015).

82. Fancy, S. P. J. et al. Parallel states of pathological Wnt signaling in neonatal brain injury and colon cancer. Nat. Neurosci. 17, 506-512 (2014).

83. Lee, H. K. et al. Daam2-PIP5K is a regulatory pathway for Wnt signaling and therapeutic target for remyelination in the CNS. Neuron 85, 1227-1243 (2015).

84. Casaccia-Bonnefil, P. et al. Oligodendrocyte precursor differentiation is perturbed in the absence of the cyclin-dependent kinase inhibitor p27Kip1. Genes Dev. 11, 2335-2346 (1997).

85. Kuypers, N. J., Bankston, A. N., Howard, R. M., Beare, J. E. \& Whittemore, S. R. Remyelinating Oligodendrocyte Precursor Cell miRNAs from the Sfmbt2 Cluster Promote Cell Cycle Arrest and Differentiation. J. Neurosci. 36, 1698-1710 (2016).

86. Magri, L. et al. c-Myc-dependent transcriptional regulation of cell cycle and nucleosomal histones during oligodendrocyte differentiation. Neuroscience 276, 72-86 (2014).

87. Magri, L. et al. E2F1 coregulates cell cycle genes and chromatin components during the transition of oligodendrocyte progenitors from proliferation to differentiation. J. Neurosci. 34, 1481-1493 (2014).

88. Hughes, E. G., Kang, S. H., Fukaya, M. \& Bergles, D. E. Oligodendrocyte progenitors balance growth with self-repulsion to achieve homeostasis in the adult brain. Nat. Neurosci. 16, 668-676 (2013).

89. Rosenberg, S. S., Kelland, E. E., Tokar, E., la Torre, De, A. R. \& Chan, J. R. The geometric and spatial constraints of the microenvironment induce oligodendrocyte differentiation. Proc. Natl. Acad. Sci. U.S.A. 105, 14662-14667 (2008).

90. Dietz, K. C., Polanco, J. J., Pol, S. U. \& Sim, F. J. Targeting human oligodendrocyte progenitors for myelin repair. Exp. Neurol. 283, 489-500 (2016). 
91. Moyon, S. et al. Functional Characterization of DNA Methylation in the Oligodendrocyte Lineage. Cell Rep 15, 748-760 (2016).

92. He, D. et al. IncRNA Functional Networks in Oligodendrocytes Reveal Stage-Specific Myelination Control by an IncOL1/Suz12 Complex in the CNS. Neuron (2016). doi:10.1016/j.neuron.2016.11.044

93. Moyon, S. et al. Efficient Remyelination Requires DNA Methylation. eNeuro 4, ENEURO.0336-16.2017 (2017).

94. Emery, B. et al. Myelin gene regulatory factor is a critical transcriptional regulator required for CNS myelination. Cell 138, 172-185 (2009).

95. Duncan, G. J. et al. Myelin regulatory factor drives remyelination in multiple sclerosis. Acta Neuropathol. 283, 330-20 (2017).

96. Ludwin, S. K. \& Maitland, M. Long-term remyelination fails to reconstitute normal thickness of central myelin sheaths. Journal of the Neurological Sciences 64, 193-198 (1984).

97. Stidworthy, M. F., Genoud, S., Suter, U., Mantei, N. \& Franklin, R. J. M. Quantifying the early stages of remyelination following cuprizone-induced demyelination. Brain Pathol. 13, 329339 (2003).

98. Powers, B. E. et al. Remyelination reporter reveals prolonged refinement of spontaneously regenerated myelin. Proc. Natl. Acad. Sci. U.S.A. 110, 4075-4080 (2013).

99. Michailov, G. V. et al. Axonal neuregulin-1 regulates myelin sheath thickness. Science 304, 700-703 (2004).

100. Brinkmann, B. G. et al. Neuregulin-1/ErbB signaling serves distinct functions in myelination of the peripheral and central nervous system. Neuron 59, 581-595 (2008).

101. Bechler, M. E., Byrne, L. \& Ffrench-Constant, C. CNS Myelin Sheath Lengths Are an Intrinsic Property of Oligodendrocytes. Curr. Biol. 25, 2411-2416 (2015).

102. Harrington, E. P. et al. Oligodendrocyte PTEN is required for myelin and axonal integrity, not remyelination. Ann. Neurol. 68, 703-716 (2010).

103. Franklin, R. J. \& Hinks, G. L. Understanding CNS remyelination: clues from developmental and regeneration biology. J. Neurosci. Res. 58, 207-213 (1999).

104. Compston, A. \& Coles, A. Multiple sclerosis. Lancet 372, 1502-1517 (2008).

105. International Multiple Sclerosis Genetics Consortium et al. Genetic risk and a primary role for cell-mediated immune mechanisms in multiple sclerosis. Nature 476, 214-219 (2011).

106. Ludwin, S. K. Understanding multiple sclerosis: lessons from pathology. Ann. Neurol. 47, 691-693 (2000).

107. Davies, C. L. \& Miron, V. E. Distinct origins, gene expression and function of microglia and monocyte-derived macrophages in CNS myelin injury and regeneration. Clin. Immunol. (2016). doi:10.1016/j.clim.2016.06.016

108. Kotter, M. R., Setzu, A., Sim, F. J., Van Rooijen, N. \& Franklin, R. J.M. Macrophage depletion impairs oligodendrocyte remyelination following lysolecithin-induced demyelination. Glia 35, 204-212 (2001).

109. Döring, A. et al. Stimulation of monocytes, macrophages, and microglia by amphotericin B and macrophage colony-stimulating factor promotes remyelination. J. Neurosci. 35, 11361148 (2015).

110. Kotter, M. R., Zhao, C., van Rooijen, N. \& Franklin, R. J. M. Macrophage-depletion induced impairment of experimental CNS remyelination is associated with a reduced oligodendrocyte progenitor cell response and altered growth factor expression. Neurobiol. Dis. 18, 166-175 (2005).

111. Robinson, S. \& Miller, R. H. Contact with central nervous system myelin inhibits oligodendrocyte progenitor maturation. Dev. Biol. 216, 359-368 (1999).

112. Trapp, B. D., Nishiyama, A., Cheng, D. \& Macklin, W. Differentiation and death of premyelinating oligodendrocytes in developing rodent brain. J. Cell Biol. 137, 459-468 (1997).

113. Plemel, J. R., Manesh, S. B., Sparling, J. S. \& Tetzlaff, W. Myelin inhibits oligodendroglial maturation and regulates oligodendrocytic transcription factor expression. Glia 61, 14711487 (2013).

114. Kotter, M. R., Li, W.-W., Zhao, C. \& Franklin, R. J. M. Myelin impairs CNS remyelination by inhibiting oligodendrocyte precursor cell differentiation. J. Neurosci. 26, 328-332 (2006).

115. Lampron, A. et al. Inefficient clearance of myelin debris by microglia impairs remyelinating 
processes. J. Exp. Med. 212, 481-495 (2015).

116. Baer, A. S. et al. Myelin-mediated inhibition of oligodendrocyte precursor differentiation can be overcome by pharmacological modulation of Fyn-RhoA and protein kinase $\mathrm{C}$ signalling. Brain 132, 465-481 (2009).

117. Skripuletz, T. et al. Astrocytes regulate myelin clearance through recruitment of microglia during cuprizone-induced demyelination. Brain 136, 147-167 (2013).

118. Psachoulia, K. et al. IL4II augments CNS remyelination and axonal protection by modulating T cell driven inflammation. Brain aww254 (2016). doi:10.1093/brain/aww254

119. Patel, J. R., McCandless, E. E., Dorsey, D. \& Klein, R. S. CXCR4 promotes differentiation of oligodendrocyte progenitors and remyelination. Proc. Natl. Acad. Sci. U.S.A. 107, 1106211067 (2010).

120. Madsen, P. M. et al. Oligodendroglial TNFR2 Mediates Membrane TNF-Dependent Repair in Experimental Autoimmune Encephalomyelitis by Promoting Oligodendrocyte Differentiation and Remyelination. J. Neurosci. 36, 5128-5143 (2016).

121. Yuen, T. J. et al. Identification of endothelin 2 as an inflammatory factor that promotes central nervous system remyelination. Brain 136, 1035-1047 (2013).

122. Miron, V. E. et al. M2 microglia and macrophages drive oligodendrocyte differentiation during CNS remyelination. Nat. Neurosci. 16, 1211-1218 (2013).

This paper provides a detailed analysis of how the innate immune response to demyelination controls the distinct stages of remyelination.

123. Rawji, K. S., Mishra, M. K. \& Yong, V. W. Regenerative Capacity of Macrophages for Remyelination. Front Cell Dev Biol 4, 47 (2016).

124. Yamasaki, R. et al. Differential roles of microglia and monocytes in the inflamed central nervous system. J. Exp. Med. 211, 1533-1549 (2014).

125. Goldmann, T. et al. Origin, fate and dynamics of macrophages at central nervous system interfaces. Nat. Immunol. 17, 797-805 (2016).

126. Bieber, A. J., Kerr, S. \& Rodriguez, M. Efficient central nervous system remyelination requires T cells. Ann. Neurol. 53, 680-684 (2003).

127. Hvilsted Nielsen, H., Toft-Hansen, H., Lambertsen, K. L., Owens, T. \& Finsen, B. Stimulation of adult oligodendrogenesis by myelin-specific T cells. Am. J. Pathol. 179, 2028-2041 (2011).

128. Dombrowski, Y. et al. Regulatory T cells promote myelin regeneration in the central nervous system. Nat. Neurosci. 485, 517 (2017).

This study reveals a pro-myelin regenerative role for the adaptive immune system, hitherto only understood in the context of its role in auto-immune mediated demyelination.

129. Goodell, M. A. \& Rando, T. A. Stem cells and healthy aging. Science 350, 1199-1204 (2015).

130. Shields, S.A., Gilson, J., Blakemore, W.F. \& Franklin, R.J.M.. Remyelination occurs as extensively but more slowly in old rats compared to young rats following fliotoxin-induced CNS demyelination. Glia 29, 102 (2000).

131. Hampton, D. W. et al. Focal Immune-Mediated White Matter Demyelination Reveals an Age-Associated Increase in Axonal Vulnerability and Decreased Remyelination Efficiency. AJPA 180, 1897-1905 (2012).

132. Pfeifenbring, S., Nessler, S., Wegner, C., Stadelmann, C. \& Brück, W. Remyelination After Cuprizone-Induced Demyelination Is Accelerated in Juvenile Mice. J. Neuropathol. Exp. Neurol. 74, 756-766 (2015).

133. Merkler, D., Ernsting, T., Kerschensteiner, M., Brück, W. \& Stadelmann, C. A new focal EAE model of cortical demyelination: multiple sclerosis-like lesions with rapid resolution of inflammation and extensive remyelination. Brain 129, 1972-1983 (2006).

134. Irvine, K. A. \& Blakemore, W. F. Age increases axon loss associated with primary demyelination in cuprizone-induced demyelination in C57BL/6 mice. J. Neuroimmunol. 175, 69-76 (2006).

135. Confavreux, C. \& Vukusic, S. Age at disability milestones in multiple sclerosis. Brain 129, 595-605 (2006).

136. Absinta, M. et al. Persistent 7-tesla phase rim predicts poor outcome in new multiple sclerosis patient lesions. J. Clin. Invest. 126, 2597-2609 (2016).

137. Goldschmidt, T., Antel, J., König, F. B., Brück, W. \& Kuhlmann, T. Remyelination capacity of the MS brain decreases with disease chronicity. Neurology 72, 1914-1921 (2009).

138. Sim, F. J., Zhao, C., Penderis, J. \& Franklin, R. J. M. The age-related decrease in CNS 
remyelination efficiency is attributable to an impairment of both oligodendrocyte progenitor recruitment and differentiation. J. Neurosci. 22, 2451-2459 (2002).

139. Doucette, J. R., Jiao, R. \& Nazarali, A. J. Age-related and cuprizone-induced changes in myelin and transcription factor gene expression and in oligodendrocyte cell densities in the rostral corpus callosum of mice. Cell. Mol. Neurobiol. 30, 607-629 (2010).

140. Woodruff, R. H., Fruttiger, M., Richardson, W. D. \& Franklin, R. J. M. Platelet-derived growth factor regulates oligodendrocyte progenitor numbers in adult CNS and their response following CNS demyelination. Mol. Cell. Neurosci. 25, 252-262 (2004).

141. Kuhlmann, T. et al. Differentiation block of oligodendroglial progenitor cells as a cause for remyelination failure in chronic multiple sclerosis. Brain 131, 1749-1758 (2008).

142. Wolswijk, G. Chronic stage multiple sclerosis lesions contain a relatively quiescent population of oligodendrocyte precursor cells. J. Neurosci. 18, 601-609 (1998).

143. Chang, A., Nishiyama, A., Peterson, J., Prineas, J. \& Trapp, B. D. NG2-positive oligodendrocyte progenitor cells in adult human brain and multiple sclerosis lesions. $J$. Neurosci. 20, 6404-6412 (2000).

This paper and ref 142 above showed that many chronic MS lesions contain sufficient aOPCs and newly-generated oligodendrocytes for remyelination. The failure of remyelination in these lesions must therefore reflect the presence of factors inhibiting the process, not a lack of cells.

144. Chari, D. M., Crang, A. J. \& Blakemore, W. F. Decline in rate of colonization of oligodendrocyte progenitor cell (OPC)-depleted tissue by adult OPCs with age. $J$. Neuropathol. Exp. Neurol. 62, 908-916 (2003).

145. Shen, S. et al. Age-dependent epigenetic control of differentiation inhibitors is critical for remyelination efficiency. Nat. Neurosci. 11, 1024-1034 (2008).

146. Natrajan, M. S. et al. Retinoid X receptor activation reverses age-related deficiencies in myelin debris phagocytosis and remyelination. Brain 138, 3581-3597 (2015).

147. Ruckh, J. M. et al. Rejuvenation of regeneration in the aging central nervous system. Cell Stem Cell 10, 96-103 (2012).

This study provides evidence that the declining efficiency of remyelination with aging (a major contributor to remyelination failure in MS) can be reversed by changing the lesion environment, the implication of which is that pharmacological approaches that target the aging progenitor are potentially effective regardless of disease stage.

148. Neves, J., Sousa-Victor, P. \& Jasper, H. Rejuvenating Strategies for Stem Cell-Based Therapies in Aging. Cell Stem Cell 20, 161-175 (2017).

149. Huang, J. K. et al. Retinoid X receptor gamma signaling accelerates CNS remyelination. Nat. Neurosci. 14, 45-53 (2011).

Using a transcription profiling approach the authors identify the nuclear receptor RXR as a potent therapeutic target for drug based enhancement of remyelination.

150. Keough, M. B. et al. An inhibitor of chondroitin sulfate proteoglycan synthesis promotes central nervous system remyelination. Nat Commun 7, 11312 (2016).

This paper draws attention to the extracellular matrix composition of demyelinated lesions as an important determinant of remyelination efficiency and how remyelination can be improved by pharmacological manipulation of the lesion ECM.

151. Siebert, J. R. \& Osterhout, D. J. The inhibitory effects of chondroitin sulfate proteoglycans on oligodendrocytes. J. Neurochem. 119, 176-188 (2011).

152. Back, S. A. et al. Hyaluronan accumulates in demyelinated lesions and inhibits oligodendrocyte progenitor maturation. Nat. Med. 11, 966-972 (2005).

153. Siebert, J. R., Stelzner, D. J. \& Osterhout, D. J. Chondroitinase treatment following spinal contusion injury increases migration of oligodendrocyte progenitor cells. Exp. Neurol. 231, 19-29 (2011).

154. Sloane, J. A. et al. Hyaluronan blocks oligodendrocyte progenitor maturation and remyelination through TLR2. Proc. Natl. Acad. Sci. U.S.A. 107, 11555-11560 (2010).

155. Lau, L. W., Cua, R., Keough, M. B., Haylock-Jacobs, S. \& Yong, V. W. Pathophysiology of the brain extracellular matrix: a new target for remyelination. Nat. Rev. Neurosci. 14, 722-729 (2013).

156. John, G. R. et al. Multiple sclerosis: re-expression of a developmental pathway that restricts oligodendrocyte maturation. Nat. Med. 8, 1115-1121 (2002).

157. Seifert, T., Bauer, J., Weissert, R., Fazekas, F. \& Storch, M. K. Notch1 and its ligand Jagged1 
are present in remyelination in a T-cell- and antibody-mediated model of inflammatory demyelination. Acta Neuropathol. 113, 195-203 (2007).

158. Stidworthy, M. F. et al. Notch1 and Jagged1 are expressed after CNS demyelination, but are not a major rate-determining factor during remyelination. Brain 127, 1928-1941 (2004).

159. Zhang, Y. et al. Notch1 signaling plays a role in regulating precursor differentiation during CNS remyelination. Proc. Natl. Acad. Sci. U.S.A. 106, 19162-19167 (2009).

160. Hu, Q.-D. et al. F3/contactin acts as a functional ligand for Notch during oligodendrocyte maturation. Cell 115, 163-175 (2003).

161. Nakahara, J., Kanekura, K., Nawa, M., Aiso, S. \& Suzuki, N. Abnormal expression of TIP30 and arrested nucleocytoplasmic transport within oligodendrocyte precursor cells in multiple sclerosis. J. Clin. Invest. 119, 169-181 (2009).

162. Mi, S. et al. LINGO-1 negatively regulates myelination by oligodendrocytes. Nat. Neurosci. 8, 745-751 (2005).

This paper first drew attention to LINGO-1 as a potential target for remyelination enhancement which subsequently led to the best developed remyelination therapy and clinical testing.

163. Mi, S. et al. Promotion of central nervous system remyelination by induced differentiation of oligodendrocyte precursor cells. Ann. Neurol. 65, 304-315 (2009).

164. Lee, X. et al. NGF regulates the expression of axonal LINGO-1 to inhibit oligodendrocyte differentiation and myelination. J. Neurosci. 27, 220-225 (2007).

165. Gautier, H. O. B. et al. Neuronal activity regulates remyelination via glutamate signalling to oligodendrocyte progenitors. Nat Commun 6, 8518 (2015).

166. Lundgaard, I. et al. Neuregulin and BDNF induce a switch to NMDA receptor-dependent myelination by oligodendrocytes. PLoS Biol. 11, e1001743 (2013).

167. Jensen, S. K. \& Yong, V. W. Activity-Dependent and Experience-Driven Myelination Provide New Directions for the Management of Multiple Sclerosis. Trends Neurosci. 39, 356-365 (2016).

168. Etxeberria, A., Mangin, J.-M., Aguirre, A. \& Gallo, V. Adult-born SVZ progenitors receive transient synapses during remyelination in corpus callosum. Nat. Neurosci. 13, 287-289 (2010).

169. Meffre, D. et al. Liver $\mathrm{X}$ receptors alpha and beta promote myelination and remyelination in the cerebellum. Proc. Natl. Acad. Sci. U.S.A. 112, 7587-7592 (2015).

170. Nelissen, K. et al. Liver X receptors regulate cholesterol homeostasis in oligodendrocytes. J. Neurosci. Res. 90, 60-71 (2012).

171. la Fuente, de, A. G. et al. Vitamin D receptor-retinoid X receptor heterodimer signaling regulates oligodendrocyte progenitor cell differentiation. J. Cell Biol. 211, 975-985 (2015).

172. Mokry, L. E. et al. Vitamin D and Risk of Multiple Sclerosis: A Mendelian Randomization Study. PLoS Med. 12, e1001866 (2015).

173. Syed, Y. A. et al. Inhibition of phosphodiesterase-4 promotes oligodendrocyte precursor cell differentiation and enhances CNS remyelination. EMBO Mol Med 5, 1918-1934 (2013).

174. Preisner, A. et al. Non-steroidal anti-inflammatory drug indometacin enhances endogenous remyelination. Acta Neuropathol. 130, 247-261 (2015).

175. Magalon, K. et al. Olesoxime accelerates myelination and promotes repair in models of demyelination. Ann. Neurol. 71, 213-226 (2012).

176. Kumar, S. et al. Estrogen receptor $\beta$ ligand therapy activates PI3K/Akt/mTOR signaling in oligodendrocytes and promotes remyelination in a mouse model of multiple sclerosis. Neurobiol. Dis. 56, 131-144 (2013).

177. Takahashi, C., Muramatsu, R., Fujimura, H., Mochizuki, H. \& Yamashita, T. Prostacyclin promotes oligodendrocyte precursor recruitment and remyelination after spinal cord demyelination. Cell Death Dis 4, e795 (2013).

178. González, G. A. et al. Tamoxifen accelerates the repair of demyelinated lesions in the central nervous system. Sci Rep 6, 31599 (2016).

179. Harsan, L.-A. et al. Recovery from chronic demyelination by thyroid hormone therapy: myelinogenesis induction and assessment by diffusion tensor magnetic resonance imaging. J. Neurosci. 28, 14189-14201 (2008).

180. Silvestroff, L., Bartucci, S., Pasquini, J. \& Franco, P. Cuprizone-induced demyelination in the rat cerebral cortex and thyroid hormone effects on cortical remyelination. Exp. Neurol. 235, 357-367 (2012). 
181. Berghoff, S. A. et al. Dietary cholesterol promotes repair of demyelinated lesions in the adult brain. Nat Commun 8, 14241 (2017).

182. Skihar, V. et al. Promoting oligodendrogenesis and myelin repair using the multiple sclerosis medication glatiramer acetate. Proc. Natl. Acad. Sci. U.S.A. 106, 17992-17997 (2009).

183. Aharoni, R. et al. Demyelination arrest and remyelination induced by glatiramer acetate treatment of experimental autoimmune encephalomyelitis. Proc. Natl. Acad. Sci. U.S.A. 105, 11358-11363 (2008).

184. Franco, P. G., Silvestroff, L., Soto, E. F. \& Pasquini, J. M. Thyroid hormones promote differentiation of oligodendrocyte progenitor cells and improve remyelination after cuprizone-induced demyelination. Exp. Neurol. 212, 458-467 (2008).

185. El-Etr, M. et al. Progesterone and nestorone promote myelin regeneration in chronic demyelinating lesions of corpus callosum and cerebral cortex. Glia 63, 104-117 (2015).

186. Blanchard, B. et al. Tocopherol derivative TFA-12 promotes myelin repair in experimental models of multiple sclerosis. J. Neurosci. 33, 11633-11642 (2013).

187. Xiao, L. et al. Diosgenin promotes oligodendrocyte progenitor cell differentiation through estrogen receptor-mediated ERK1/2 activation to accelerate remyelination. Glia 60, 10371052 (2012).

188. Cadavid, D. et al. Safety and efficacy of opicinumab in acute optic neuritis (RENEW): a randomised, placebo-controlled, phase 2 trial. Lancet Neurol 16, 189-199 (2017).

189. Lucchinetti, C. et al. Heterogeneity of multiple sclerosis lesions: implications for the pathogenesis of demyelination. Ann. Neurol. 47, 707-717 (2000).

190. Lucchinetti, C. et al. A quantitative analysis of oligodendrocytes in multiple sclerosis lesions. A study of 113 cases. Brain 122 ( Pt 12), 2279-2295 (1999).

191. Mei, F. et al. Micropillar arrays as a high-throughput screening platform for therapeutics in multiple sclerosis. Nat. Med. 20, 954-960 (2014).

This paper describes an innovative platform for screening for potential remyelination enhancing agents, and led to the identification of clemastine, currently in clinical trials

192. Najm, F. J. et al. Drug-based modulation of endogenous stem cells promotes functional remyelination in vivo. Nature 522, 216-220 (2015).

This paper reported a remyelination screening platform based on mouse epiblast-derived OPCs to identify two FDA-approved compounds, miconazole and clobestol, that enhance remyelination.

193. Deshmukh, V. A. et al. A regenerative approach to the treatment of multiple sclerosis. Nature 502, 327-332 (2013).

This study used another high throughput screening platform to reveal antagonism of muscarinic receptors as a pharmacological means of potentially enhancing remyelination.

194. Eleuteri, C. et al. A staged screening of registered drugs highlights remyelinating drug candidates for clinical trials. Sci Rep 7, 45780 (2017).

195. Mei, F. et al. Identification of the Kappa-Opioid Receptor as a Therapeutic Target for Oligodendrocyte Remyelination. J. Neurosci. 36, 7925-7935 (2016).

196. Zuchero, J. B. et al. CNS myelin wrapping is driven by actin disassembly. Dev. Cell 34, 152167 (2015).

197. Lachapelle, F. et al. Transplantation of CNS fragments into the brain of shiverer mutant mice: extensive myelination by implanted oligodendrocytes. I. Immunohistochemical studies. Dev. Neurosci. 6, 325-334 (1983).

The first demonstration that transplanted oligodendrocytes could form new myelin, using a naturally-occurring mouse mutant that lacks normal myelin as a result of the loss of myelin basic protein, a protein essential for the compaction of the myelin sheath

198. Blakemore, W. F. \& Crang, A. J. The use of cultured autologous Schwann cells to remyelinate areas of persistent demyelination in the central nervous system. Journal of the Neurological Sciences 70, 207-223 (1985).

199. Blakemore, W. F. \& Franklin, R. J. Transplantation of glial cells into the CNS. Trends Neurosci. 14, 323-327 (1991).

200. Wang, S. et al. Human iPSC-derived oligodendrocyte progenitor cells can myelinate and rescue a mouse model of congenital hypomyelination. Cell Stem Cell 12, 252-264 (2013).

201. Windrem, M. S. et al. Fetal and adult human oligodendrocyte progenitor cell isolates myelinate the congenitally dysmyelinated brain. Nat. Med. 10, 93-97 (2004).

202. Windrem, M. S. et al. Neonatal chimerization with human glial progenitor cells can both 
remyelinate and rescue the otherwise lethally hypomyelinated shiverer mouse. Cell Stem Cell 2, 553-565 (2008).

203. Gupta, N. et al. Neural stem cell engraftment and myelination in the human brain. Sci Transl Med 4, 155ra137-155ra137 (2012).

204. Piao, J. et al. Human embryonic stem cell-derived oligodendrocyte progenitors remyelinate the brain and rescue behavioral deficits following radiation. Cell Stem Cell 16, 198-210 (2015).

205. Harrisingh, M. C. \& Ffrench-Constant, C. Can the irradiated brain be salvaged by oligodendrocyte precursor transplantation? Cell Stem Cell 16, 113-114 (2015).

206. Koyanagi-Aoi, M. et al. Differentiation-defective phenotypes revealed by large-scale analyses of human pluripotent stem cells. Proc. Natl. Acad. Sci. U.S.A. 110, 20569-20574 (2013).

207. Douvaras, P. \& Fossati, V. Generation and isolation of oligodendrocyte progenitor cells from human pluripotent stem cells. Nat Protoc 10, 1143-1154 (2015).

208. Kerschensteiner, M. et al. Targeting experimental autoimmune encephalomyelitis lesions to a predetermined axonal tract system allows for refined behavioral testing in an animal model of multiple sclerosis. AJPA 164, 1455-1469 (2004).

209. Mallik, S., Samson, R. S., Wheeler-Kingshott, C. A. M. \& Miller, D. H. Imaging outcomes for trials of remyelination in multiple sclerosis. J. Neurol. Neurosurg. Psychiatr. jnnp-2014307650 (2014). doi:10.1136/jnnp-2014-307650

210. Absinta, M., Sati, P. \& Reich, D. S. Advanced MRI and staging of multiple sclerosis lesions. Nat Rev Neurol 12, 358-368 (2016).

211. van den Elskamp, I. J. et al. Lesional magnetization transfer ratio: a feasible outcome for remyelinating treatment trials in multiple sclerosis. Mult. Scler. 16, 660-669 (2010).

212. Turati, L. et al. In vivo quantitative magnetization transfer imaging correlates with histology during de- and remyelination in cuprizone-treated mice. NMR Biomed 28, 327-337 (2015).

213. Brown, R. A., Narayanan, S. \& Arnold, D. L. Segmentation of magnetization transfer ratio lesions for longitudinal analysis of demyelination and remyelination in multiple sclerosis. Neuroimage 66, 103-109 (2013).

214. Stankoff, B. et al. Imaging central nervous system myelin by positron emission tomography in multiple sclerosis using [methyl- $\left.{ }^{11} \mathrm{C}\right]-2-(4$-'methylaminophenyl)- 6-hydroxybenzothiazole. Ann. Neurol. 69, 673-680 (2011).

215. Bodini, B. et al. Dynamic imaging of individual remyelination profiles in multiple sclerosis. Ann. Neurol. 79, 726-738 (2016).

216. Leocani, L., Rocca, M. A. \& Comi, G. MRI and neurophysiological measures to predict course, disability and treatment response in multiple sclerosis. Curr. Opin. Neurol. 29, 243-253 (2016).

217. Connick, P. et al. Autologous mesenchymal stem cells for the treatment of secondary progressive multiple sclerosis: an open-label phase 2a proof-of-concept study. Lancet Neurol 11, 150-156 (2012).

218. Franklin R.J.M., et al Endogenous remyelination in the CNS. In: MYELIN REPAIR AND Neuroprotection. (Eds. Duncan ID, Franklin RJM). Springer. pp 71-92. (2012) 
Figure 1 | The logic of promoting remyelination. Following demyelination, which in the autoimmune disease multiple sclerosis is consequent to the pathological activation of $T$ cells and macrophages, the myelin sheath is lost but the underlying axon remains intact. This enables the naturally occurring regenerative response of remyelination to generate new sheaths from newly formed oligodendrocytes. Existing oligodendrocytes whose sheaths have been damaged do not contribute to the regenerative process. In the absence of remyelination, energy efficient conduction cannot be restored and the supportive role of the myelin is lost. This leads to energy deficiency, perturbed axonal transport (as illustrated by the accumulation of mitochondria at the node) and ultimately axonal degeneration. This degeneration can trigger a secondary inflammatory response, as illustrated by the presence of activated macrophages around the degenerating axon.

Figure 2 | The biology of remyelination. a | Following damage to myelinated areas in the CNS (illustrated in the upper left panel by a representation of a coronal section through a human brain affected by multiple sclerosis). remyelination is initiated by activation of oligodendrocyte progenitor cells (OPCs; upper right panel). These become activated (as represented by the colour change), divide and form new oligodendrocytes. Both progenitor cells within and around the lesion can contribute, with the latter migrating into the lesion after activation as shown on the right side of the panel. Note the presence of macrophages in the lesion; as discussed in the main text, macrophages play essential roles in the phagocytosis of myelin debris and the promotion of the regenerative response. Following oligodendrocyte differentiation, myelin formation proceeds in three steps as shown in the sequence illustrated in the lower panel: the formation of multiple processes and the expression of myelin proteins such as myelin basic protein, the initial wrapping of the axon by an elaboration of myelin membrane and, finally, the formation of multi-layered and compacted sheaths by the continued elaboration of membrane, further wrapping of the axon and extrusion of the cytoplasm. $\mathbf{b}$ | Genetic fate mapping studies, in which fluorescent marker proteins are expressed exclusively within adult progenitors, have revealed how these cells give rise to new remyelinating oligodendrocytes. The left-hand panel shows a cross section from an adult mouse spinal cord in which many of the OPCs are green. These cells are especially concentrated in focal areas of demyelination induced 6 days previously by injection of lysolecithin into the left ventral white matter (dotted line), indicative of the recruitment phase of remyelination. The right-hand panel shows a similar lesion 21 days after lesion induction when all of the demyelinated axons are fully remyelinated. The white arrows indicate new myelin sheaths expressing myelin proteolipid protein (red), which have been made by the green OPCs that have differentiated into myelin-forming oligodendrocytes (from REF 39).

Figure 3 | The architecture of remyelination. a | The sheaths formed by remyelination are frequently thinner than those around axons myelinated during development. A hypothetical model to explain this characteristic feature of remyelination is illustrated in the upper panel. Studies showing that oligodendrocytes can form sheaths around artificial fibers of diameters equivalent to axons reveal the existence of an intrinsic pathway requiring only an appropriate shape to form a sheath (upper left panel). Myelin plasticity triggered by activity and changes in axonal diameter (so called adaptive 
myelination) then results in the elaboration of further myelin membrane, leading to thickening and lengthening of the sheath. After this sheath is lost by disease in the adult CNS, when the axon shape is no longer changing (lower right panel) it is the intrinsic pathway in the newly-formed oligodendrocyte that is responsible for remyelination - as a result the sheath is thinner than those present around unaffected axons (lower left panel). b | Electronmicrograph of myelinated and remyelinated axons following ethidium bromide-induced demyelination the deep cerebellar white matter of an adult rat. The myelin sheath thickness of the myelinated axons $\left(\mathrm{M}_{1}\right.$ and $\left.\mathrm{M}_{2}\right)$ is proportional to the axon diameter. The remyelinated axons can be recognised by the relatively thin myelin sheaths $\mathrm{R}_{1}$ and $\mathrm{R}_{2}$ ), which are uniformly thin regardless of the axon diameter. Thus, remyelination is readily identified in larger diameter axons, while for small-diameter axons myelinated and remyelinated becomes difficult to distinguish. $\mathbf{c}$ | The g ratio is used to quantify the relationship between the axon diameter $(x)$ and the myelinated axon $(y)$ : the thinner the myelin sheath, the higher the $g$ ratio, and hence remyelinated axons have $g$ ratios that are higher than those of myelinated axons (with the exception of the small diameter axons). d | In developmental myelination, there is an increase in myelin sheath thickness with increasing diameter of axons. In remyelination, however, the myelin sheath thickness remains the same regardless of the diameter (see $R_{1}$ with $R_{2}$ in part $b$ ). (B-D are adapted from figures in REF 218).

Figure 4 | Drug discovery for remyelination. The different steps of oligodendrocyte formation and differentiation that might be targeted are shown in the top panel, with progenitor cells on the left and myelinating oligodendrocytes on the right. A number of screens of FDA-approved drugs have been performed, which have revealed a number of drugs such as those listed here that are potential remyelination medicines. These screens have targeted the oligodendrocyte differentiation step or, in one case, the process of initial wrapping using micropillars, as illustrated. None of these screening platforms have to date targeted the final critical stage of myelin sheath formation, and it remains unknown whether additional signals will be required for this process or whether promoting differentiation will suffice. 


\section{Glossary terms}

G ratio: this term describes the ratio of the axon circumference to the circumference of the myelinated axon and is used to provide a quantitative measure of the myelin sheath thickness compared to the axon diameter: in remyelination the $g$ ratio is usually increased.

Demyelination: This is the pathological process in which myelin sheaths are lost form axons that remain intact. It is sometimes called primary demyelination to distinguish it from loss of myelin that is secondary to axonal loss, which is more accurately called Wallerian degeneration and should not be called demyelination.

Remyelination: This is the regenerative process involving the generation of new oligodendrocytes from CNS resident progenitor cells and their reinvestment of new myelin sheaths around the demyelinated axon.

Oligodendrocyte: This is the cell that makes myelin in the central nervous system. A single oligodendrocyte can make up to 80 separate myelin sheaths, although around $10-20$ is a more usual number.

Schwann cell: This is the cell that make myelin in the peripheral nervous system. A single Schwann cell only ever makes a single myelin sheath. In certain circumstances, Schwann cells can remyelinate demyelinated axons in the central nervous system.

Multiple Sclerosis: This is a common autoimmune-mediated disease of the central nervous system characterised by multiple acute inflammatory foci involving immune-mediated demyelination which can undergo spontaneous remyelination but with disease progression this becomes less efficient leaving axons chronically demyelinated and prone to irreversible degeneration.

Leucodystrophies: These are a family of genetic disease usually characterised by inadequate myelination or demyelination. 